\title{
Ethyl Acetate Extracts of Bushen-Yizhi Formula Attenuates Aging-Induced Dementia through Suppressing NLRP3 Inflammasome Activation in SAMP8 Mice
}

\section{Shi-Jie Su}

Guangzhou University of Chinese Medicine

Wei-Wu Cai

Guangzhou University of Chinese Medicine Hao-Fei Liu

Guangzhou University of Chinese Medicine

Shi-Jie Zhang ( $\nabla$ shijiezhang@gzucm.edu.cn )

Guangzhou University of Chinese Medicine

Qi Wang

Guangzhou University of Chinese Medicine

Si-Jun Liu

Guangzhou University of Chinese Medicine

\section{Research}

Keywords: BSYZ-E, aging, dementia, NLRP3 inflammasome, SAMP8 mice

Posted Date: November 9th, 2020

DOl: https://doi.org/10.21203/rs.3.rs-102344/v1

License: (c) (i) This work is licensed under a Creative Commons Attribution 4.0 International License.

Read Full License 


\section{Abstract}

Background: Similar features indicated common regulators between aging and aging-induced dementia, which demonstrated profound correlations with each other. However, the mechanism remains unclear. Bushen-Yizhi Formula(BSYZ-F) has been used for managing dementia in the clinic for hundreds of years. In this study, we aimed to further explore the mechanism of athyl acetate extracts of BSYZ-F(BSYZ-E) attenuates aging-induced dementia in Senescence-Accelerated Mouse Prone 8 (SAMP8) mice.

Methods: As an age-related model, SAMP8 mice have been assigned to receive the corresponding interventions in this study. The Morris water maze was used to evaluate the learning and memory ability in SAMP8 mice. The marker level of oxidative stress(ROS and SOD) and nitrosative stress(NO and iNOS) were measured using commercial kits. The quantification of inflammation-related proteins (NLRP3, ASC, Caspase-1, IL-1 $\beta$, and IL-18) and related apoptotic proteins (Bax, Bcl-2, Caspase-3, and Cleaved-Caspase3 ) in hippocampal tissue were measured using Western blot.

Results: In behavioral, the Morris Water Maze test demonstrated BSYZ-E alleviated the cognitive impairments in SAMP8 mice. Mechanism studies indicated that BSYZ-E increased the vitality of SOD, declined the validity of NO, iNOS, and MDA in SAMP8 mice. Besides, BSYZ-E relieved neuronal apoptosis by regulating the expression of related apoptotic proteins (Bax, Bcl-2, Caspase-3, and Cleaved-Caspase3) and inhibited NLRP3 inflammasome activation by inflammation-related proteins (NLRP3, ASC, Caspase-1, IL-1 $\beta$, and IL-18) in SAMP8 mice.

Conclusions: These results suggested that BSYZ-E attenuates aging-induced dementia by suppressing the activation of NLRP3 inflammasome in SAMP8 mice.

\section{Background}

Aging is a multifactorial and irreversible process that deteriorates organism homeostasis and resilience[1]. In decades, increasing evidence has shown abnormal age-related changes in dementia underlying structures and functions[2-4]. Between 2015 and 2050, more than $56 \%$ of older people will increased, which leads to a higher risk of dementia epidemic $[5,6]$. The clinical manifestation of dementia is cognitive decline[7]. NLRP3 inflammasomes have been regarded as indispensable section, which triggers pathological neuroinflammation in animal models of dementia[8]. NLRP3 inflammasome induced microglia-mediated neuroinflammation, which caused hippocampal-dependent learning and memory deficits[9]. NLRP3 inflammasome inhibitor alleviated cognitive deficits and $\beta$-amyloid pathology in APP/PS1 mice[10, 11].

Bushen-Yizhi Formula (BSYZ-F), an herbal formula of Traditional Chinese Medicine (TCM), is made of Cnidium monnieri (L.) Cusson, Paeonia $\times$ suffruticosa Andrews, Panax quinquefolius L, Fallopia multiflora (Thunb.) Harald, Lycium Chinese Mill, Ligustrum lucidum W.T.Aiton. BSYZ-F has been used for managing dementia in the clinic because it could improve the mini-mental state examination score in dementia patients[12]. Previous studies demonstrated that BSYZ-F regulated not only the cholinergic 
signaling pathway, nerve growth factor pathway, and apoptosis pathway in the ibotenic acid-treated rat[13], but also anti-oxidative stress and anti-apoptosis in SCOP-induced mice[14]. Meanwhile, the compounds of the ethyl acetate extract components (BSYZ-E), an active extracts of Bushen-Yizhi formula, were identified in our previous study[15]. BSYZ-E had been reported that it has the effects of antiapoptosis, anti-oxidative stress in SCOP-induced mice.

As a model of age-induced cognitive deficits, Senescence-Accelerated Mouse Prone 8 (SAMP8) mice exhibited age-accelerated learning and memory disorders[16]. Therefore, we employed SAMP8 mice for exploring whether BSYZ-E could ameliorate aging-induced cognitive dysfunction. Our results suggested that BSYZ-E alleviates aging-induced dementia via suppressing NLRP3 inflammasome activation in SAMP8 mice.

\section{Materials And Methods}

\section{Arrangement of ethyl acetate extract}

BSYZ-F consists of Cnidium monnieri (L.) Cusson, Paeonia $\times$ suffruticosa Andrews, Panax quinquefolius L, Fallopia multiflora (Thunb.) Harald, Lycium Chinese Mill, Ligustrum lucidum W.T.Aiton, and the six herbs were mixed in a proportion of 3:3:2:2:2:2. Chemical decomposition of BSYZ-E was analyzed by HPLC in our previous study[17]. BSYZ-E was provided by the Institute of Tropical Medicine, Guangzhou University of Chinese Medicine (GZUCM, Guangzhou, China). Drug certificate (NO.20140425) was issued by GZUCM. According to the grams per kilogram of body weight, $1 \mathrm{~g}$ BSYZ-E represented $113.76 \mathrm{~g}$ of original herbs.

\section{Animals and housing}

Seven-month-old male SAMP8 mice and age-matched Senescence-Accelerated Resistant 1 (SAMR1) mice were purchased from the Center Laboratory Animal, Peking University (Beijing, China). All mice were arranged at the Center Experimental Animal of GZUCM under suitable temperature, humidity, and light conditions. All Animals were fed ad libitum. The Animal Ethics Committee approved experimental protocols of GZUCM, and experiments were performed in compliance with relative protocols.

\section{Administration of BSYZ-E}

SAMP8 mice were assigned randomly to five groups, depending on the experimental protocol: SAMP8 group (vehicle control), BSYZ-E L group (1.46 g/ kg/day), BSYZ-E M group (2.92 g/kg/day), and BSYZ-E H group ( $5.84 \mathrm{~g} / \mathrm{kg} /$ day), Donepezil (Eisai China Inc) group (3 mg/kg/day). SAMR1 group, regarded as healthy controls, received saline only. All animals were treated with gavage BSYZ-E, Donepezil, or saline once daily for 30 days continuously.

\section{Morris Water Maze (MWM)Test}

From 31 to 37 days of the experiment, the MWM test was performed as previous[18], with the purpose of appraise spatial learning memory capability in different groups. The apparatus used for the MWM test, 
designing by the round pond $(120 \mathrm{~cm}$ in diameter) containing opaque water, adding a non-toxic white ink (temperature: $26 \pm 1^{\circ} \mathrm{C}$ ) with $30 \mathrm{~cm}$ depth. During the acquisition phase, the hidden platform with a $10 \mathrm{~cm}$ diameter is hidden $1 \mathrm{~cm}$ below the center of the target quadrant. The mice were subjected to a position navigation test 4 times each day for five days. The time (escape latency) and swimming path of finding the hidden platform were recorded. If mice were unable to reach the hidden platform after sixty seconds, swimming time was calculated as $60 \mathrm{sec}$, and then they were navigated to the hidden platform to learn for $20 \mathrm{~s}$. On day seven, the platform will be removed. At the same time, the mice were arranged to swim for $60 \mathrm{~s}$ starting from the identical point. The time spent on the target quadrant and crossing times of the platform location was recorded to appraise the cognitive ability of the SAMP8 mice.

\section{Western blotting analysis}

Hippocampus was homogenized in pre-cooling RIPA lysis buffer $\left(4^{\circ} \mathrm{C}, 5 \mathrm{~min}, 3000 \mathrm{~g}\right)$. Then homogenates were centrifuged $\left(4^{\circ} \mathrm{C}, 15 \mathrm{~min}, 12000 \mathrm{~g}\right)$, and the supernatants were obtained. The supernatant of protein concentration was determined and corresponding protein was separated between different groups with SDS PAGE gel. Protein was shifted to the PVDF membranes and blocked with $5 \%$ BSA for 90 min at $37^{\circ} \mathrm{C}$. Incubated the protein-containing PVDF membrane with the target antibody overnight at $4{ }^{\circ} \mathrm{C}$ and washed again for $5 \mathrm{~min}$, four times with $1 \times$ TBST. The cleaned PVDF membrane was incubated with the target secondary antibody (anti-mouse and anti-rabbit) for one hour at $37^{\circ} \mathrm{C}$. The membrane was washed in $1 \times$ TBST 4 times again for $5 \mathrm{~min} /$ time. Target protein bands were imaged using enhanced chemiluminescence reagent (ECL).

\section{Measurement of MDA, SOD, NO, and iNOS}

Hippocampus was homogenized with pre-cooling $0.9 \%$ saline and centrifuged $\left(4{ }^{\circ} \mathrm{C}, 5 \mathrm{~min}, 3000 \mathrm{~g}\right)$. The supernatants were used for detecting the MDA, SOD, NO, and iNOS, based on the manufacturer's protocols. Their respective absorbance was measured $(532 \mathrm{~nm}, 560 \mathrm{~nm}, 550 \mathrm{~nm}$, and $530 \mathrm{~nm}$, respectively) by using Universal Microplate Spectrophotometer.

\section{Statistical analysis}

Experimental data are presented as mean \pm standard error of the mean (SEM). All data were statistically analyzed by using SPSS 19.0 statistical software (IBM, Endicott, NY). Followed by Dunnett's post-hoc test, the differences between groups were analyzed using ANOVA (One-way analysis of variance and Two-way analysis of variance). Comparative differences were deemed too significant at $p$-values $<0.05$.

\section{Results}

BSYZ-E alleviates the spatial learning memory impairments in SAMP8 mice.

As one of the validating behavioral, the MWM test measures to appraise spatial learning memory[19, 20]. As illustrated (Fig. 1), the action of BSYZ-E in spatial learning memory impairments were observed in SAMP8 mice via the MWM test. Throughout five days of training, the mice consecutively shortened the time of finding the hidden platforms. The escape latency in the SAMP8 group was markedly longer, 
compared with the SAMR1 group. After treating with BSYZ-E and Donepezil, SAMP8 mice in these groups spent less time in finding the target, compared with the mice in the SAMP8 group (Fig. 1A). we removed the hidden platform on the seventh day in order to permit different groups swimming randomly to appraise their spatial learning and memory., The SAMP8 group had lesser crossing times and took less time on the target platform compared to the SAMR1 group. Treating with both BSYZ-E and Donepezil groups (Fig. 1B-C) had a greater crossing times and more time in the objective platform compared to the SAMP8 group. The consequence demonstrated that the mice in the SAMP8 group had minor alternations, compared with those in the SAMR1 group. After treating with BSYZ-E and Donepezil, the proportion of alternation was considerably enhanced when contrasted with the SAMP8 group.

\section{BSYZ-E modifies oxidative stress in the hippocampus of SAMP8 mice.}

The MDA levels and SOD vitality in the hippocampus was assessed, to dictate the fact of BSYZ-E on antioxidative stress Compared to the SAMR1 group, the MDA level notably heightened in the brain tissue of the SAMP8 group. After treatment, the MDA level was downregulated in the SAMP8 mice treated by BSYZ-E, in contrast with the SAMP8 group (Fig. 2). Besides, the SOD vitality of the hippocampus in the SAMR1 group was remarkably higher than other groups. After the treatment of BSYZ-E or Donepezil, the vitality of SOD has greatly downregulated when compared to the SAMP8 group. Treatment of mice with BSYZ-E H or Donepezil significantly improved SOD vitality compared with the vehicle control group.

\section{BSYZ-E ameliorates nitrosative stress in the hippocampus of SAMP8 mice.}

The production of NO and iNOS was estimated to evaluate the action of BSYZ-E in SAMP8 mice (Fig. 3). The NO level was greatly expanded in the SAMP8 group compared to the SAMR1 group. After treating by BSYZ-E or Donepezil, NO level of the hippocampus in SAMP8 mice was remarkably decreased in all treatment groups compared with the SAMR1 group, but not back to normal levels. Interestingly, BSYZ-E $(1.46-5.84 \mathrm{~g} / \mathrm{kg} /$ day) reduced NO levels in dose gradient dependence. Besides, compared to the SAMR1 group, the SAMP8 group has higher expression of iNOS vitality in the hippocampus of the SAMP8 mice. Treating the SAMP8 mice with BYSZ-E M, BSYZ-E H, and Donepezil considerably decreased the production of iNOS, in contrast with the SAMP8 group. The effect of BSYZ-E H (5.84 g/ kg/day) was more remarkable than that of the low and middle dose level of BSYZ-E.

\section{BSYZ-E relieves neuronal apoptosis in the hippocampus of SAMP8 mice.}

The expression of apoptosis-associated proteins was analyzed by western blot, to further study the mechanism of neuronal apoptosis in the hippocampus of SAMP8 mice, In the SAMP8 group, the protein of Bax and Cleaved-Caspase-3 has higher expression, but lower expression in Bcl-2 protein, compared to the SAMR1 group (Fig. 4). After treatment with BSYZ-E and Donepezil, the expression of Bax and CleavedCaspase-3 severely downregulated, but Bcl-2 protein expression remarkably upregulated. The higher dose of BSYZ-E in the hippocampus had more significant expression results which demonstrated that BSYZ-E has the function of alleviating neuronal apoptosis. 


\section{BSYZ-E inhibits NLRP3 inflammasome activation in the hippocampus of SAMP8 mice.}

Western blotting was used to detect the expression of related inflammatory proteins in the NLRP3 inflammasome signaling pathway. The results showed (Fig. 5) that the expression of NLRP3, ASC, Caspase-1, IL-18, and IL-1 $\beta$ increased in the hippocampus of SAMP8 mice, compared to the SAMR1 group. It proves that the hippocampal NLRP3 inflammasome in SAMP8 mice has been activated. However, the expression of these proteins was deregulated in the hippocampus of SAMP8 mice after treating with BSYZ-E and Donepezil. As the dose of BSYZ-E increased, the effect became more pronounced. It confirmed that BSYZ-E suppressed the activation of NLRP3 inflammasome in SAMP8 mice.

\section{Discussion}

We illustrated that BSYZ-E could advance age-related cognitive decline in SAMP8 mice in this study. BSYZ-E modified oxidative stress and nitrosative stress, as well as reduced neuronal apoptosis and neuroinflammation.

Advanced age is always a primary hazard of neurodegenerative diseases[21, 22]. Accumulating evidence suggested that the risk factor of dementia is attributed to age-related neuropathologies[23,24]. The main clinical manifestation of aging-induced dementia is cognitive impairment and decline[25]. The mechanisms of aging-induced dementia are associated with oxidative stress, neuronal apoptosis, and neuroinflammation[26]. As a neuropathological animal model, SAMP8 mice have been universally used for researching accelerated brain-aging and dementia $[27,28]$. In this study, SAMP8 mice displayed distinct age-related deterioration in cognitive ability and relative pathological features. Therefore, SAMP8 mice were deemed as an experimental animal model to investigate whether BSYZ-E could attenuate the behavioural and pathological of aging-induced dementia in this study. In previous studies, BSYZ-F has good efficacy for the treatment of clinical dementia[12]. We also demonstrated that BSYZ-F/BSYZ-E could improve learning and memory impairment through different signal pathways in different animal models[29, 30]. In our study, we further studied the mechanism of BSYZ-E alleviating neuroinflammation in SAMP8 mice. The MWM test was designed to appraise the cognitive ability in SAMP8 mice. The result indicated that BSYZ-E could improve cognitive impairments in SAMP8 mice.

Produced by complicated processes, reactive oxygen/nitrogen species formation promotes cellular senescence and organismal aging[31]. The relation of oxidative/nitrosative stress and accelerated-aging is well established[32, 33]. Reactive oxygen species (ROS) overproduction contributes to activate the proinflammatory pathways[34], which promotes neuroinflammation in aging-induced dementia [35, 36]. Superoxide dismutase (SOD) and Malondialdehyde (MDA), as the oxidative stress-related biomarkers, indicated the overproduction of ROS. Vivo aging model showed an increase in nitrosative stress with high expression of nitric oxide (NO) and inducible nitric oxide synthase (iNOS)[37]. In this study, the application of BSYZ-E and Donepezil can decrease MDA levels and increase SOD vitality in SAMP8 mice. Besides, NO levels and iNOS vitality in SAMP8 mice have been downregulated under the treatment of 
BSYZ-E and Donepezil. These results manifested the efficiency of BSYZ-E on alleviating oxidative and nitrosative stress in SAMP8 mice.

Apoptosis, a programmed cell death, leads to a vicious circle of neurodegenerative diseases like Alzheimer's disease[38]. Neuronal degeneration is well known as a primary cause leading to memory loss in dementia[39]. Aging can promote neural apoptosis by regulating the activation of Caspase cascades and BAD (Bcl-2-associated death promoter)[40]. Bcl-2 is an anti-apoptosis protein that could inhibit neuronal apoptosis, and $\mathrm{Bax}$ is a homolog of $\mathrm{Bcl}-2$ that could promote apoptosis[41]. The pro-apoptotic protein Bax activation relevant to the intrinsic mitochondrial apoptotic pathway, followed by Caspase-3 activated, leading to cell death[42]. The results revealed the expression of apoptotic protein (Bax, Caspase-3, and Cleaved-Caspase-3) prominently declined, yet related anti-apoptosis protein Bcl-2 express greatly increased after the treatment of BSYZ-E and Donepezil. It demonstrated that BSYZ-E distinctly decreased neural apoptosis in SAMP8 mice.

Inflammasomes, as a group of large multimeric complex proteins, pertain to a large family of intracellular pattern recognition receptors[43, 44]. NLRP3 (NOD-, LRR- and pyrin domain-containing 3) inflammasome, consisting of NLRP3, ASC, Caspase-1, is a pivotal element of the innate immune system. NLRP3 inflammasome mediated Caspase- 1 activation, pyrolysis as well as promoted the secretion of proinflammatory cytokines interleukin-18 (IL-18)/IL-1 $\beta[45-47]$. Multiple endogenous or exogenous danger signals, including infection and metabolic dysfunction, trigger the inflammatory reaction initiated by the NLRP3 inflammasome[48]. Inhibition of NLRP3 activation can improve cognitive deficits, including agedependent cognitive impairment[11,49,50]. The main components of BSYZ-E are analyzed by HPLC in our previous study[17]. We found that the main components of BSYZ-E include osthole and emodin. Osthole improved the cognitive function of vascular dementia rats via inhibition NLRP3 inflammasome[51]. Emodin exerted anti-inflammatory effects by relieving NLRP3 inflammasome activation[52], whose signaling has correlated with the neuroinflammation[53]. Massive experiments have determined that the expression of NLRP3 inflammasome-mediated neuroinflammation can be assembled via elevating the expression of NLRP3, ASC, Caspase-1, IL-18, and IL-1 $\beta$ in the hippocampus[44, 54]. In our study, we discovered higher expression inflammatory-related proteins, including NLRP3, ASC, Caspase-1, IL-1 $\beta$, and IL-18 in the hippocampus of SAMP8 mice than SAMR1 mice, which confirmed that NLRP3 inflammasome activation was recognized in SAMP8 mice. Neuroinflammation was reduced by treating with BSYZ-E and Donepezil, which indicated that BSYZ-E could protect against the NLRP3 inflammasome-mediated neuroinflammation in SAMP8 mice. Hence, we demonstrated the effect of BSYZ-E on alleviating NLRP3 inflammasome-mediated neuroinflammation in SAMP8 mice.

\section{Conclusion}

The study elaborated that BSYZ-E could ameliorate neuroinflammation through inhibiting NLRP3 inflammasome activation, which could be a promising dementia therapeutics. Nonetheless, the active ingredients of BSYZ-E need further analysis, and the further pharmacological mechanism of BSYZ-E is still needed to be elucidated. 


\section{Abbreviations}

TCM Traditional Chinese Medicine

BSYZ-F Bushen-Yizhi Formula

BSYZ-E ethyl acetate extract components of Bushen-Yizhi Formula

SAMP8 Senescence-Accelerated Mouse Prone 8 mice

SAMR1 Senescence-Accelerated Resistant 1 mice

MWM Morris Water Maze

ECL enhanced chemiluminescence

SEM mean \pm standard error of the mean

ANOVA Analysis of Variance

SOD Superoxide dismutase

MDA Malondialdehyde

ROS Reactive oxygen species

NO nitric oxide

iNOS inducible nitric oxide synthase

NLRP3 NOD-, LRR- and pyrin domain-containing 3

IL-18 interleukin-18

IL-1 $\beta$ interleukin-1 $\beta$

\section{Declarations}

\section{Ethics approval and consent to participate}

The Animal Ethics Committee approved experimental protocols of Guangzhou University of Chinese Medicine, and experiments were performed in compliance with relative protocols.

\section{Consent to publish}

All of authors consent to publication of this work in Chinese Medicine. 
Not applicable.

\section{Competing interests}

The authors declare that they have no competing interests.

\section{Funding}

This work was supported by Key laboratory project of colleges and universities in Guangdong province (No. 2019KSYS005), Guangzhou Science Technology and Innovation Commission Technology Research Projects (No. 201805010005) and Guangdong Provincial Key Laboratory of Research on Emergency in TCM (2017B030314176).

\section{Authors' Contributions}

Shi-Jie Zhang designed the experiments. Shi-Jie Su, Wei-Wu Cai, Si-Jun Liu and Qi Wang carrried to most of the experiments. Shi-Jie Su and Shi-Jie Zhang wrote the manuscript. Si-Jun Liu and Qi Wang modified manuscript.

\section{Acknowledgements}

Not applicable.

\section{References}

1. López-Otín C, et al. The hallmarks of aging. Cell. 2013;153(6):1194-217.

2. Lambourne SL, et al. Increased tau phosphorylation on mitogen-activated protein kinase consensus sites and cognitive decline in transgenic models for Alzheimer's disease and FTDP-17: evidence for distinct molecular processes underlying tau abnormalities. Mol Cell Biol. 2005;25(1):278-93.

3. Schmitt K, Grimm A, Eckert A. Amyloid- $\beta$-Induced Changes in Molecular Clock Properties and Cellular Bioenergetics. Front Neurosci. 2017;11:124.

4. Yang T, et al. The impact of cerebrovascular aging on vascular cognitive impairment and dementia. Ageing Res Rev. 2017;34:15-29.

5. Sensi SL. Alzheimer's Disease, time to turn the tide. Aging. 2018;10(10):2537-8.

6. Crous-Bou M, et al. Alzheimer's disease prevention: from risk factors to early intervention. Alzheimers Res Ther. 2017;9(1):71.

7. Rabin LA, Smart CM, Amariglio RE. Subjective Cognitive Decline in Preclinical Alzheimer's Disease. Annu Rev Clin Psychol. 2017;13:369-96.

8. White CS, et al. Inflammasomes as therapeutic targets for Alzheimer's disease. Brain Pathol. 2017;27(2):223-34.

9. Wang D, et al. The role of NLRP3-CASP1 in inflammasome-mediated neuroinflammation and autophagy dysfunction in manganese-induced, hippocampal-dependent impairment of learning and memory ability. Autophagy. 2017;13(5):914-27. 
10. Tejera $D$, et al. Systemic inflammation impairs microglial $A \beta$ clearance through NLRP3 inflammasome. Embo j. 2019;38(17):e101064.

11. Dempsey $C$, et al. Inhibiting the NLRP3 inflammasome with MCC950 promotes non-phlogistic clearance of amyloid- $\beta$ and cognitive function in APP/PS1 mice. Brain Behav Immun. 2017;61:30616.

12. Wang H, Lai S, Sun J. [Ethological examination of Alzheimer's disease model rats treated with bushen yizhi decoction]. Zhongguo Zhong Xi Yi Jie He Za Zhi. 2000;20(10):771-3.

13. Hou X-Q, et al. Alleviating Effects of Bushen-Yizhi Formula on Ibotenic Acid-Induced Cholinergic Impairments in Rat. Rejuvenation Research. 2015;18(2):111-27.

14. Hou X-Q, et al. Bushen-Yizhi formula ameliorates cognition deficits and attenuates oxidative stressrelated neuronal apoptosis in scopolamine-induced senescence in mice. Int $\mathrm{J}$ Mol Med. 2014;34(2):429-39.

15. Zhang S-J, et al., Ethyl Acetate Extract Components of Bushen-Yizhi Formula Provides Neuroprotection against Scopolamine-induced Cognitive Impairment. Scientific Reports, 2017. 7.

16. Liu B, Liu J, Shi JS. SAMP8 Mice as a Model of Age-Related Cognition Decline with Underlying Mechanisms in Alzheimer's Disease. J Alzheimers Dis. 2020;75(2):385-95.

17. Zhang SJ, et al. Ethyl Acetate Extract Components of Bushen-Yizhi Formula Provides Neuroprotection against Scopolamine-induced Cognitive Impairment. Sci Rep. 2017;7(1):9824.

18. Zhang S-J, et al. Bushen-Yizhi formula ameliorates cognitive dysfunction through SIRT1/ER stress pathway in SAMP8 mice. Oncotarget. 2017;8(30):49338-50.

19. Vorhees CV, Williams MT. Assessing spatial learning and memory in rodents. ILAR journal. 2014;55(2):310-32.

20. Vorhees CV, Williams MT. Value of water mazes for assessing spatial and egocentric learning and memory in rodent basic research and regulatory studies. Neurotoxicol Teratol. 2014;45:75-90.

21. Chi H, Chang HY, Sang TK. Neuronal Cell Death Mechanisms in Major Neurodegenerative Diseases. Int J Mol Sci, 2018. 19(10).

22. Hou Y, et al. Ageing as a risk factor for neurodegenerative disease. Nat Rev Neurol. 2019;15(10):56581.

23. Boyle PA, et al. Attributable risk of Alzheimer's dementia attributed to age-related neuropathologies. Ann Neurol. 2019;85(1):114-24.

24. Power MC, et al. Combined neuropathological pathways account for age-related risk of dementia. Ann Neurol. 2018;84(1):10-22.

25. DeTure MA, Dickson DW. The neuropathological diagnosis of Alzheimer's disease. Mol Neurodegener. 2019;14(1):32.

26. Sharma P, et al. Comprehensive review of mechanisms of pathogenesis involved in Alzheimer's disease and potential therapeutic strategies. Prog Neurobiol. 2019;174:53-89. 
27. Griñán-Ferré $C$, et al. Understanding Epigenetics in the Neurodegeneration of Alzheimer's Disease: SAMP8 Mouse Model. J Alzheimers Dis. 2018;62(3):943-63.

28. Akiguchi I, et al. SAMP8 mice as a neuropathological model of accelerated brain aging and dementia: Toshio Takeda's legacy and future directions. Neuropathology. 2017;37(4):293-305.

29. Hou XQ, et al. Alleviating effects of Bushen-Yizhi formula on ibotenic acid-induced cholinergic impairments in rat. Rejuvenation Res. 2015;18(2):111-27.

30. Hou XQ, et al. Bushen-Yizhi formula ameliorates cognition deficits and attenuates oxidative stressrelated neuronal apoptosis in scopolamine-induced senescence in mice. Int J Mol Med. 2014;34(2):429-39.

31. Liguori I, et al. Oxidative stress, aging, and diseases. Clin Interv Aging. 2018;13:757-72.

32. Singh A, et al., Oxidative Stress: A Key Modulator in Neurodegenerative Diseases. Molecules, 2019. 24(8).

33. Maurya PK, et al. The role of oxidative and nitrosative stress in accelerated aging and major depressive disorder. Prog Neuropsychopharmacol Biol Psychiatry. 2016;65:134-44.

34. Kim YK, et al. The role of pro-inflammatory cytokines in neuroinflammation, neurogenesis and the neuroendocrine system in major depression. Prog Neuropsychopharmacol Biol Psychiatry. 2016;64:277-84.

35. Banks WA, et al. Lipopolysaccharide-induced blood-brain barrier disruption: roles of cyclooxygenase, oxidative stress, neuroinflammation, and elements of the neurovascular unit. J Neuroinflammation. 2015;12:223.

36. Ma Y, et al., Flavonoid-Rich Ethanol Extract from the Leaves of Diospyros kaki Attenuates DGalactose-Induced Oxidative Stress and Neuroinflammation-Mediated Brain Aging in Mice. Oxid Med Cell Longev, 2018. 2018: p. 8938207.

37. Bellaver B, et al. Hippocampal Astrocyte Cultures from Adult and Aged Rats Reproduce Changes in Glial Functionality Observed in the Aging Brain. Mol Neurobiol. 2017;54(4):2969-85.

38. Shalini S, et al. Old, new and emerging functions of caspases. Cell Death Differ. 2015;22(4):526-39.

39. Li X, et al. Human Neural Stem Cell Transplantation Rescues Cognitive Defects in APP/PS1 Model of Alzheimer's Disease by Enhancing Neuronal Connectivity and Metabolic Activity. Front Aging Neurosci. 2016;8:282.

40. Yang TY, et al., The Effect of Bornyl cis-4-Hydroxycinnamate on Melanoma Cell Apoptosis Is Associated with Mitochondrial Dysfunction and Endoplasmic Reticulum Stress. Int J Mol Sci, 2018. 19(5).

41. Obulesu M, Lakshmi MJ. Apoptosis in Alzheimer's disease: an understanding of the physiology, pathology and therapeutic avenues. Neurochem Res. 2014;39(12):2301-12.

42. Vince JE, et al. The Mitochondrial Apoptotic Effectors BAX/BAK Activate Caspase-3 and - 7 to Trigger NLRP3 Inflammasome and Caspase-8 Driven IL-1 $\beta$ Activation. Cell Rep. 2018;25(9):233953.e4. 
43. Rathinam VA, Fitzgerald KA. Inflammasome Complexes: Emerging Mechanisms Effector Functions Cell. 2016;165(4):792-800.

44. Man SM, Kanneganti TD. Regulation of inflammasome activation. Immunol Rev. 2015;265(1):6-21.

45. Ozaki E, Campbell M, Doyle SL. Targeting the NLRP3 inflammasome in chronic inflammatory diseases: current perspectives. J Inflamm Res. 2015;8:15-27.

46. Kelley N, et al., The NLRP3 Inflammasome: An Overview of Mechanisms of Activation and Regulation. Int J Mol Sci, 2019. 20(13).

47. He Y, Hara H, Núñez G. Mechanism and Regulation of NLRP3 Inflammasome Activation. Trends Biochem Sci. 2016;41(12):1012-21.

48. Zhou R, et al. A role for mitochondria in NLRP3 inflammasome activation. Nature. 2011;469(7329):221-5.

49. Fan Y, et al. Inhibiting the NLRP3 Inflammasome With MCC950 Ameliorates Isoflurane-Induced Pyroptosis and Cognitive Impairment in Aged Mice. Front Cell Neurosci. 2018;12:426.

50. Ward R, et al. NLRP3 inflammasome inhibition with MCC950 improves diabetes-mediated cognitive impairment and vasoneuronal remodeling after ischemia. Pharmacol Res. 2019;142:237-50.

51. Liu Y, et al. Osthole Improves Cognitive Function of Vascular Dementia Rats: Reducing A Deposition via Inhibition NLRP3 Inflammasome. Biol Pharm Bull. 2020;43(9):1315-23.

52. Han JW, et al. Anti-inflammatory effect of emodin via attenuation of NLRP3 inflammasome activation. Int J Mol Sci. 2015;16(4):8102-9.

53. Venegas C, Heneka MT. Danger-associated molecular patterns in Alzheimer's disease. J Leukoc Biol. 2017;101(1):87-98.

54. Elliott El, Sutterwala FS. Initiation and perpetuation of NLRP3 inflammasome activation and assembly. Immunol Rev. 2015;265(1):35-52.

\section{Figures}



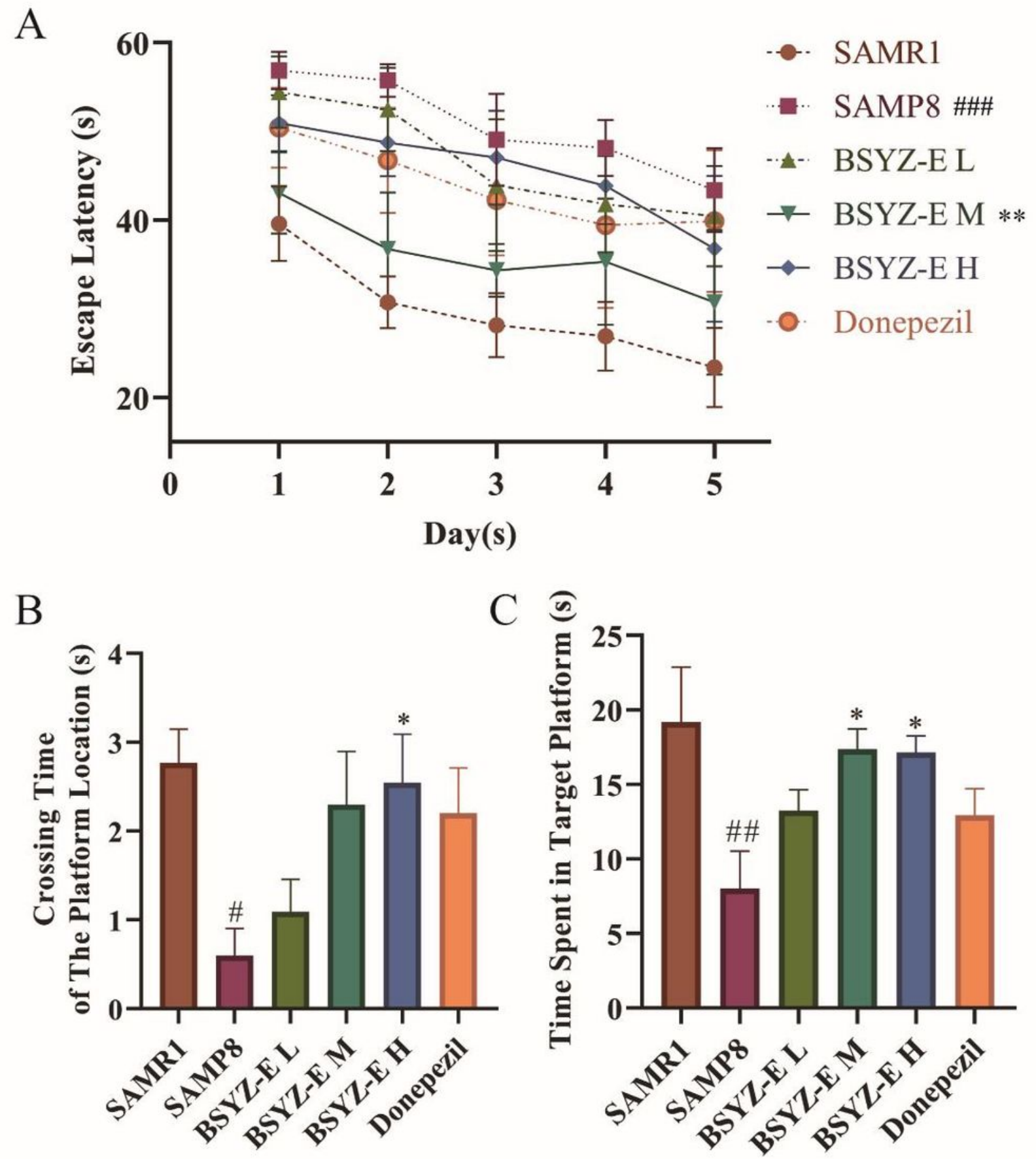

Figure 1

BSYZ-E alleviates the spatial learning memory impairments in SAMP8 mice. (A) Escape latencies in the Morris Water Maze test during the positioning navigation test. (B) Time spent in the target platform. (C) The number of times crossing the platform location during the probe trial. BSYZ-E L (1.46 $/ \mathrm{kg} /$ day); BSYZ-E M (2.92 g/kg/day); BSYZ-E H ( $5.84 \mathrm{~g} / \mathrm{kg} /$ day). Experimental values were expressed as means \pm SEM. $\# p<0.05, \# \# p<0.01$ versus SAMR1 group. ${ }^{*} p<0.05,{ }^{* \star} p<0.01,{ }^{* \star *} \mathrm{p} \otimes 0.001$ versus SAMP8 group. 

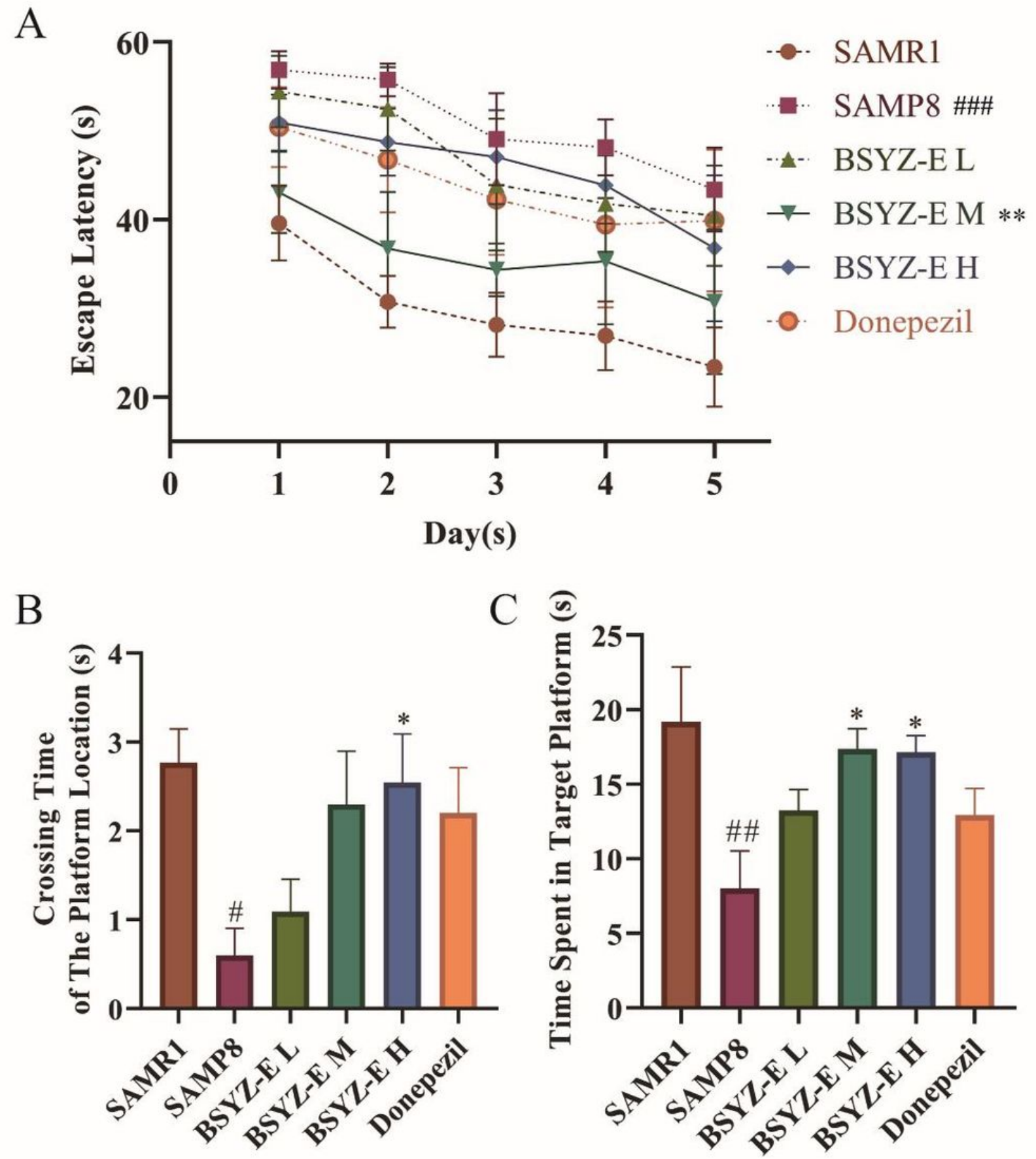

Figure 1

BSYZ-E alleviates the spatial learning memory impairments in SAMP8 mice. (A) Escape latencies in the Morris Water Maze test during the positioning navigation test. (B) Time spent in the target platform. (C) The number of times crossing the platform location during the probe trial. BSYZ-E L (1.46 $/ \mathrm{kg} /$ day); BSYZ-E M (2.92 g/kg/day); BSYZ-E H ( $5.84 \mathrm{~g} / \mathrm{kg} /$ day). Experimental values were expressed as

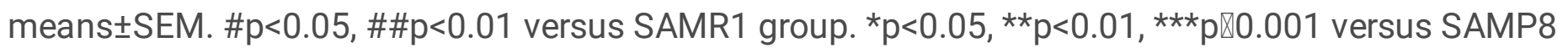
group. 

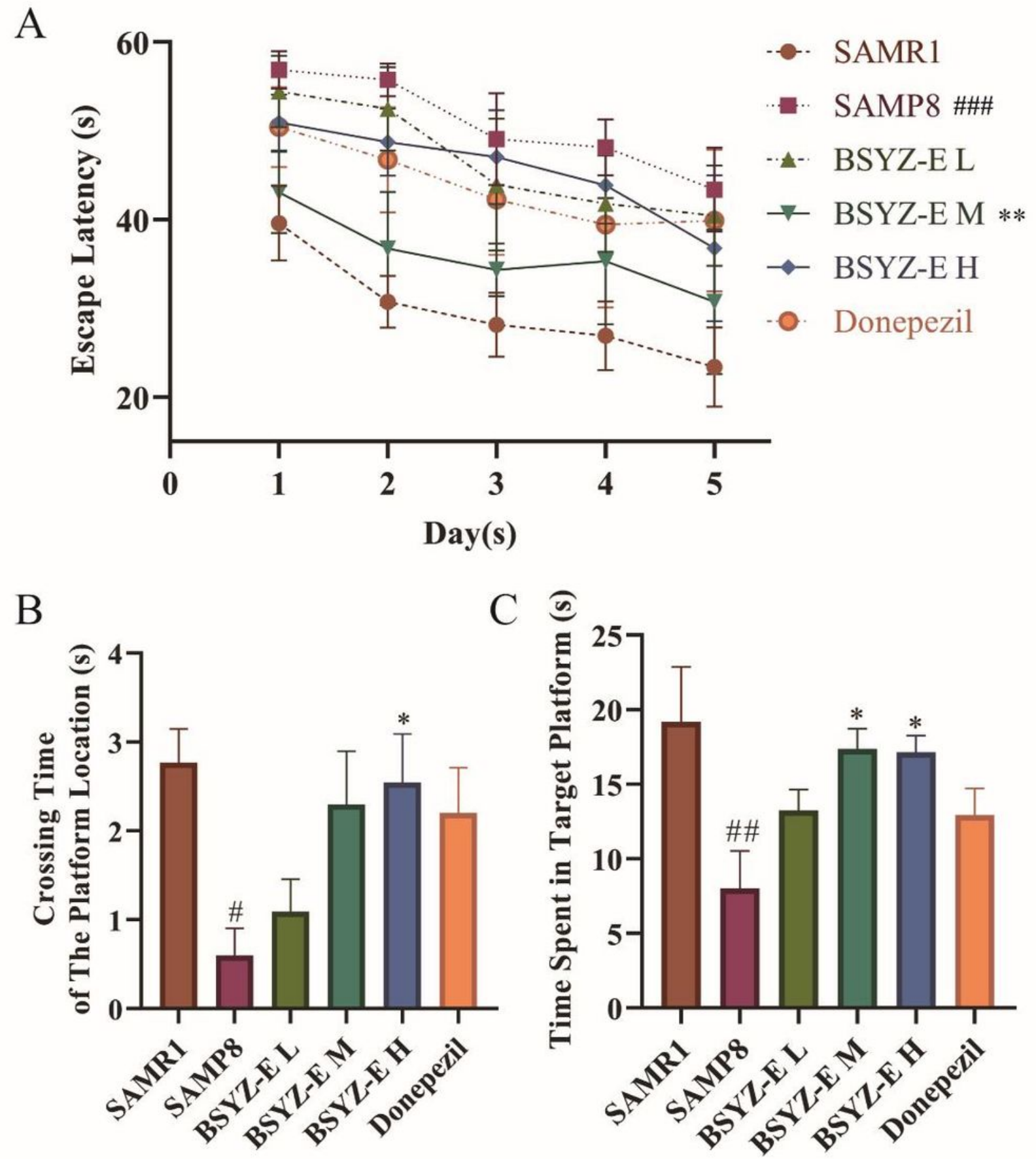

Figure 1

BSYZ-E alleviates the spatial learning memory impairments in SAMP8 mice. (A) Escape latencies in the Morris Water Maze test during the positioning navigation test. (B) Time spent in the target platform. (C) The number of times crossing the platform location during the probe trial. BSYZ-E L (1.46 $/ \mathrm{kg} /$ day); BSYZ-E M (2.92 g/kg/day); BSYZ-E H ( $5.84 \mathrm{~g} / \mathrm{kg} /$ day). Experimental values were expressed as

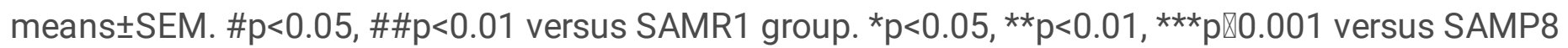
group. 
A

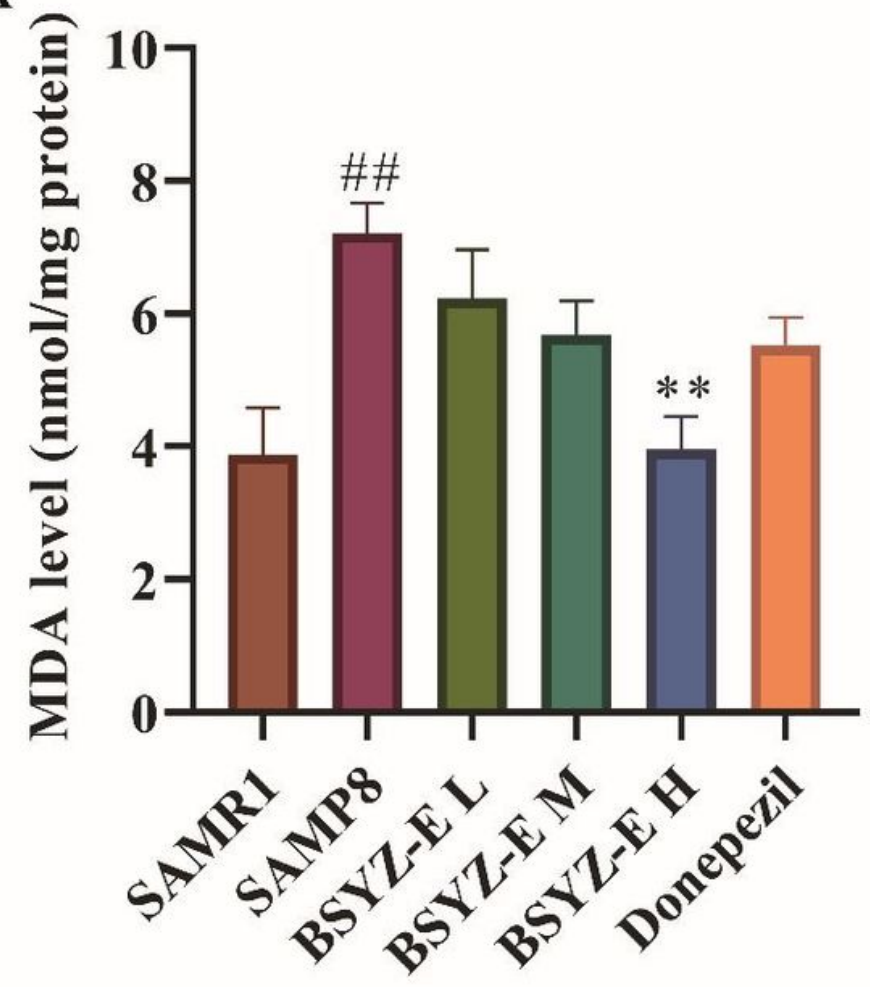

B

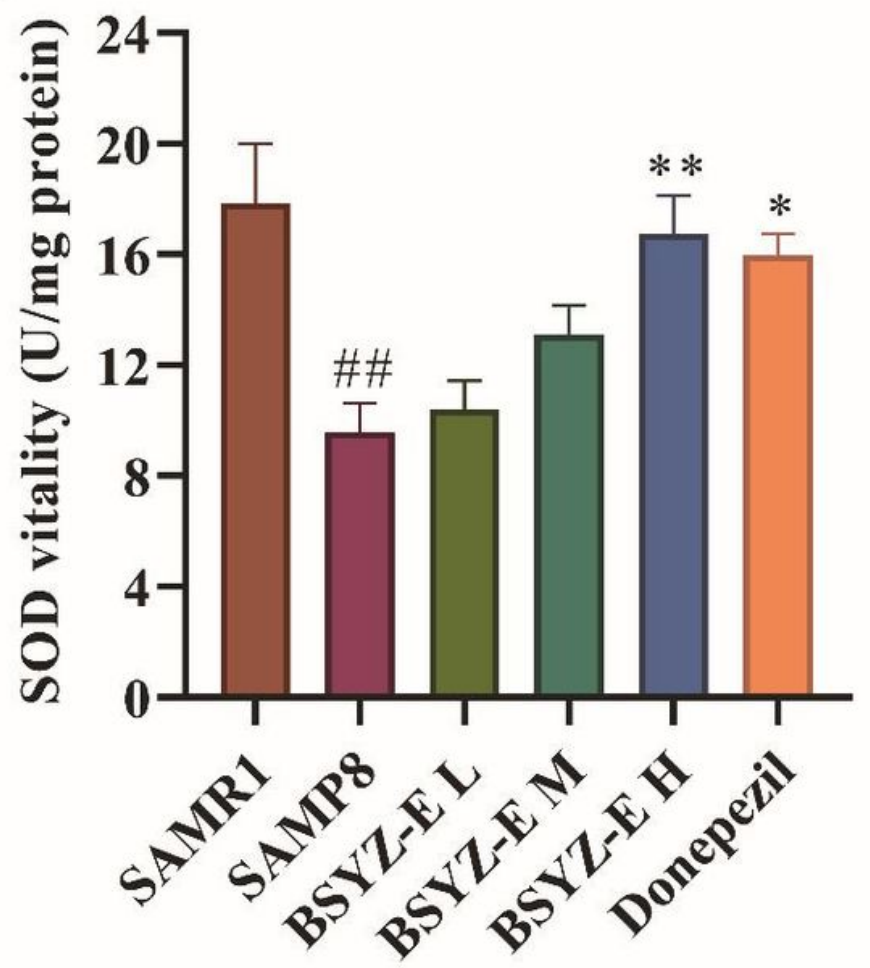

Figure 2

BSYZ-E modifies oxidative stress in the hippocampus of SAMP8 mice. (A) SOD vitality, (B) MDA level were measured in the hippocampus. BSYZ-E L (1.46g/kg/day); BSYZ-E M (2.92 g/kg/day); BSYZ-E H $(5.84 \mathrm{~g} / \mathrm{kg} /$ day $)$. Experimental values were expressed as means $\pm S E M . \# p<0.05, \# \# p<0.01$ versus SAMR 1 group. ${ }^{*} \mathrm{p}<0.05,{ }^{* \star} \mathrm{p}<0.01,{ }^{\star \star *} \mathrm{p} \otimes 0.001$ versus SAMP8 group. 
A

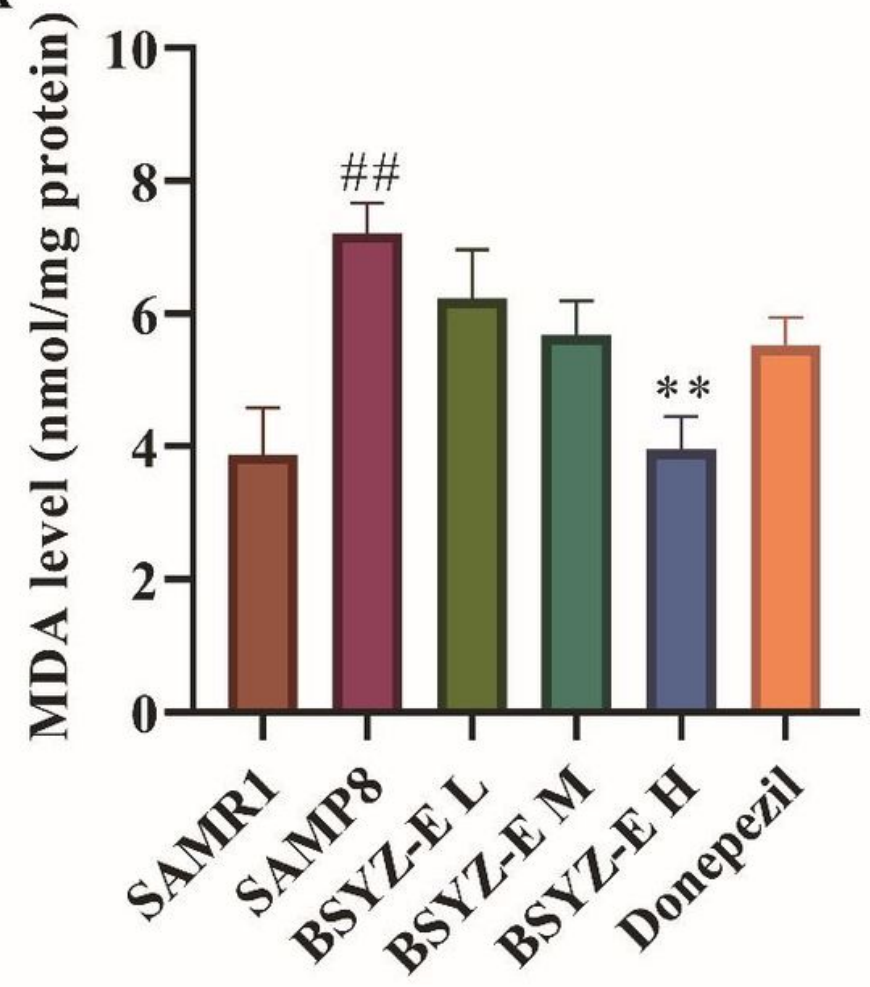

B

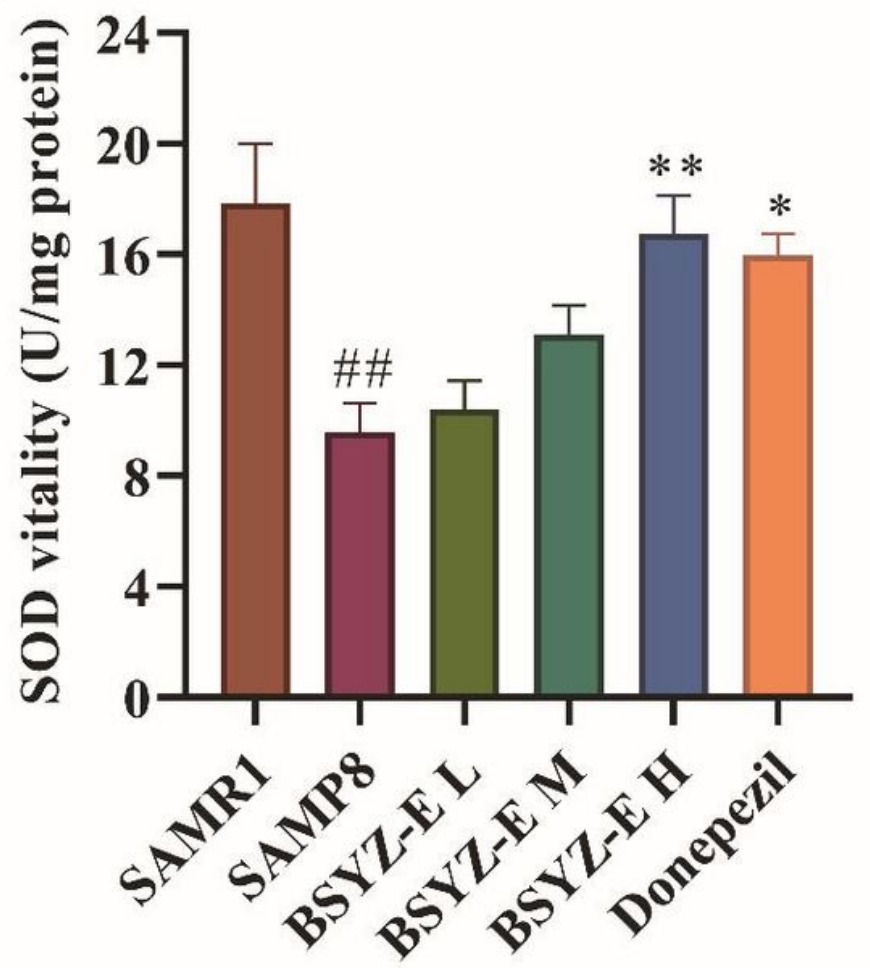

Figure 2

BSYZ-E modifies oxidative stress in the hippocampus of SAMP8 mice. (A) SOD vitality, (B) MDA level were measured in the hippocampus. BSYZ-E L (1.46g/kg/day); BSYZ-E M (2.92 g/kg/day); BSYZ-E H $(5.84 \mathrm{~g} / \mathrm{kg} /$ day $)$. Experimental values were expressed as means $\pm S E M . \# p<0.05, \# \# p<0.01$ versus SAMR 1 group. ${ }^{*} \mathrm{p}<0.05,{ }^{* \star} \mathrm{p}<0.01,{ }^{\star \star *} \mathrm{p} \otimes 0.001$ versus SAMP8 group. 
A

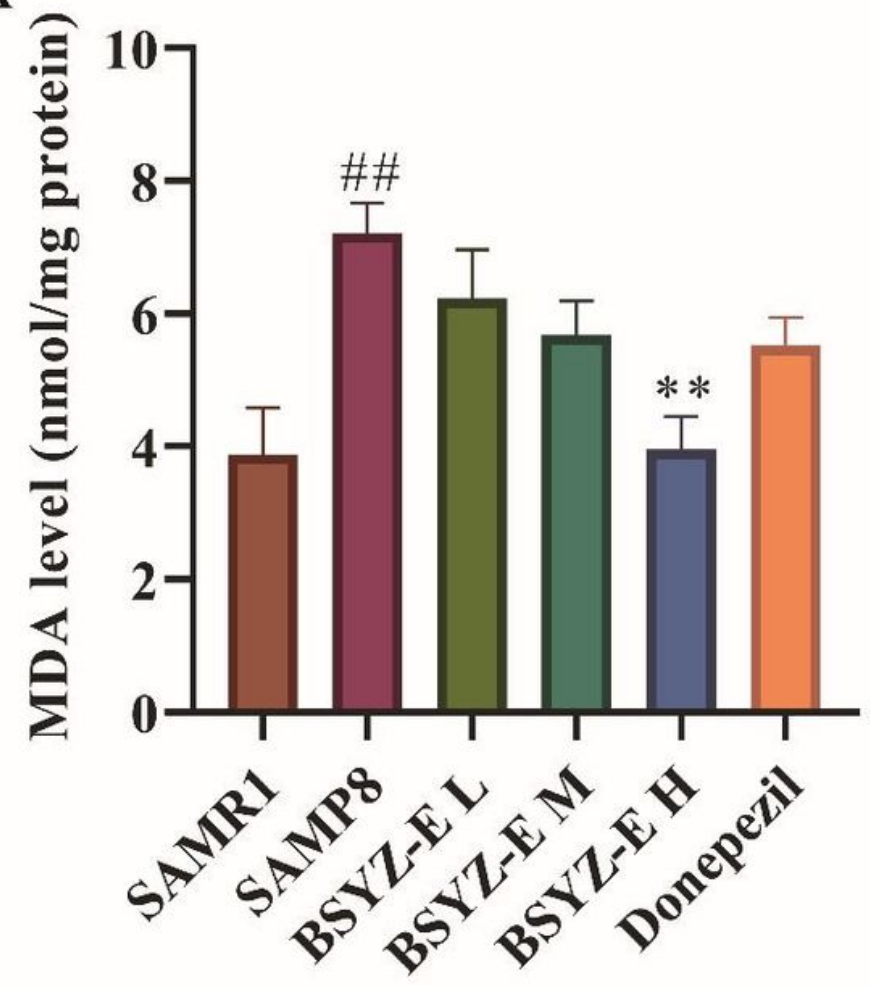

B

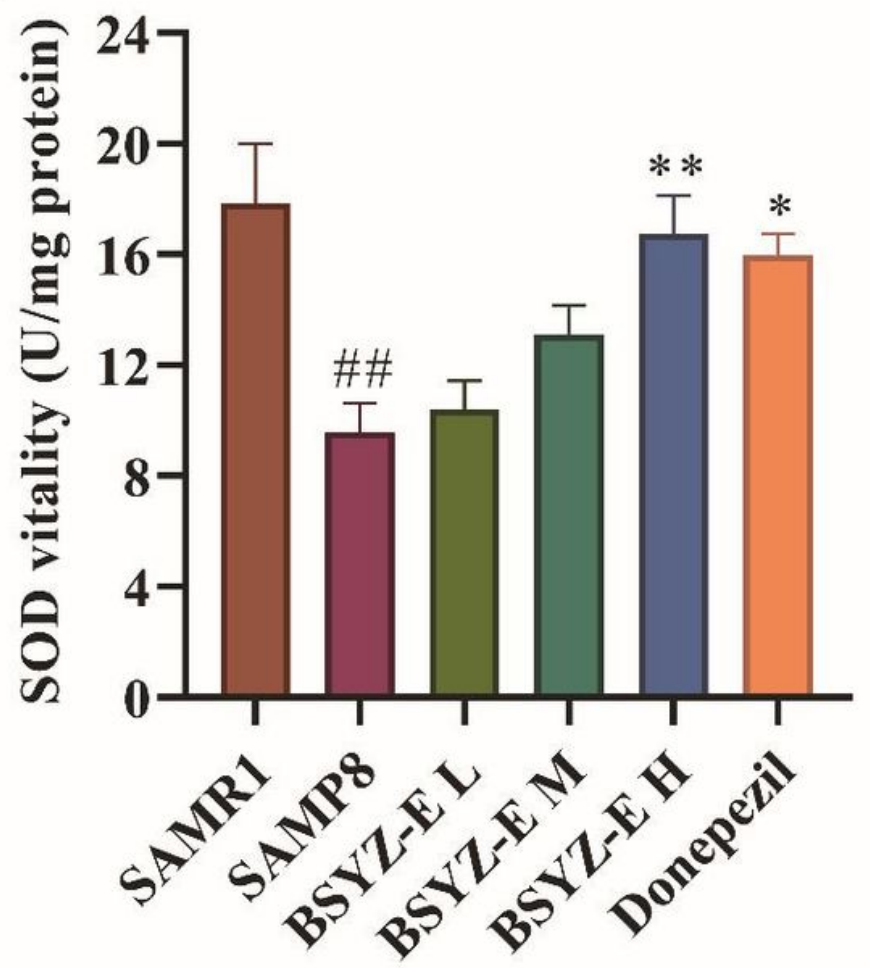

Figure 2

BSYZ-E modifies oxidative stress in the hippocampus of SAMP8 mice. (A) SOD vitality, (B) MDA level were measured in the hippocampus. BSYZ-E L (1.46g/kg/day); BSYZ-E M (2.92 g/kg/day); BSYZ-E H $(5.84 \mathrm{~g} / \mathrm{kg} /$ day $)$. Experimental values were expressed as means $\pm S E M . \# p<0.05, \# \# p<0.01$ versus SAMR 1 group. ${ }^{*} \mathrm{p}<0.05,{ }^{* \star} \mathrm{p}<0.01,{ }^{\star \star *} \mathrm{p} \otimes 0.001$ versus SAMP8 group. 
A

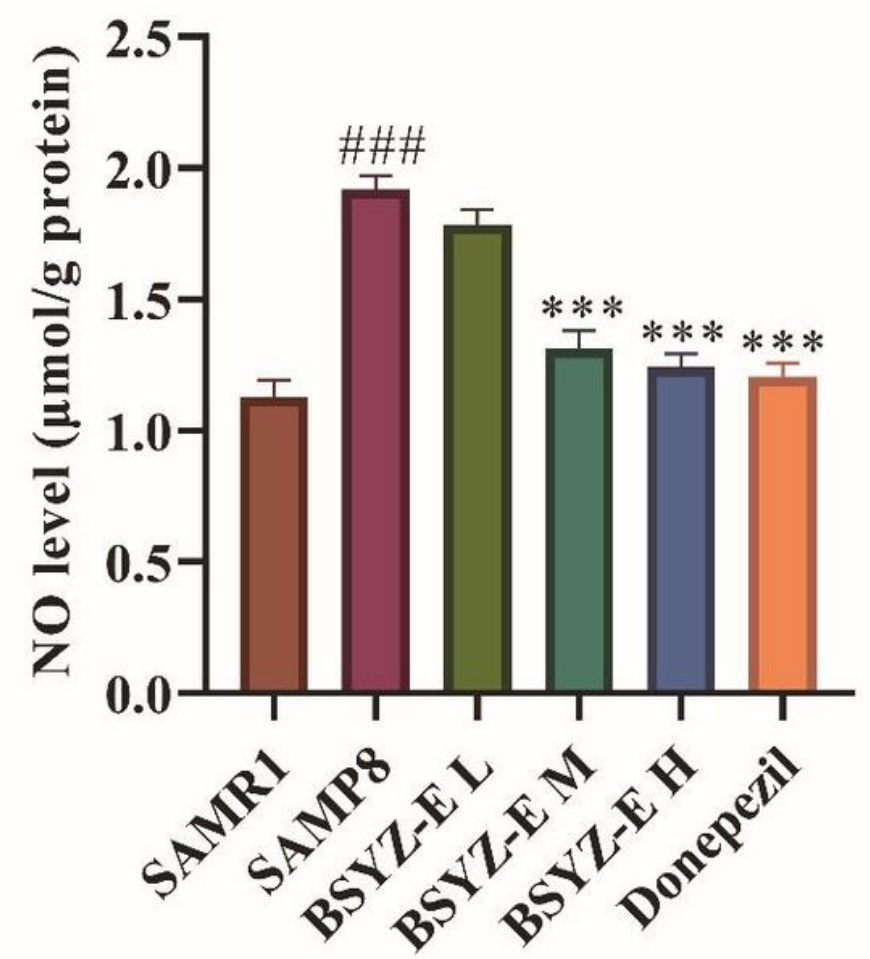

B

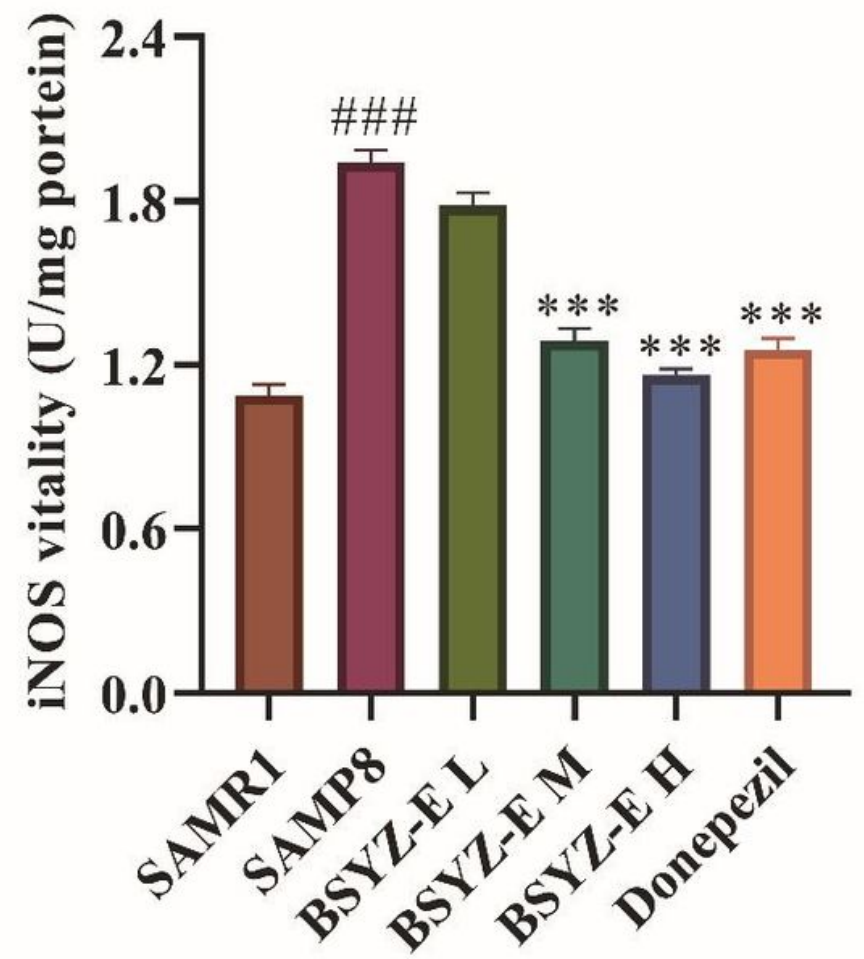

Figure 3

BSYZ-E ameliorates nitrosative stress in the hippocampus of SAMP8 mice. (A) NO level, (B) iNOS vitality were measured in the hippocampus. BSYZ-E L (1.46g/kg/day); BSYZ-E M (2.92 g/kg/day); BSYZ-E H $(5.84 \mathrm{~g} / \mathrm{kg} /$ day $)$. Experimental values were expressed as means $\pm S E M . \# p<0.05, \# \# p<0.01$ versus SAMR 1 group. ${ }^{*} \mathrm{p}<0.05,{ }^{* \star} \mathrm{p}<0.01,{ }^{\star \star *} \mathrm{p} \otimes 0.001$ versus SAMP8 group. 
A

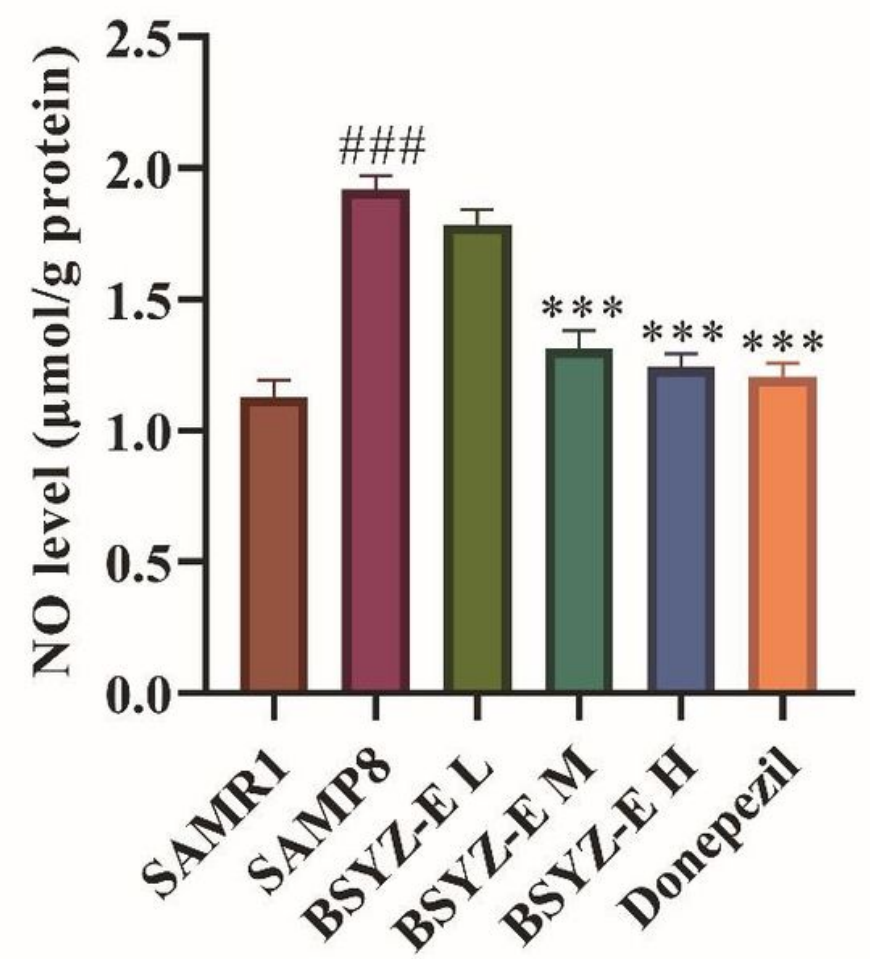

B

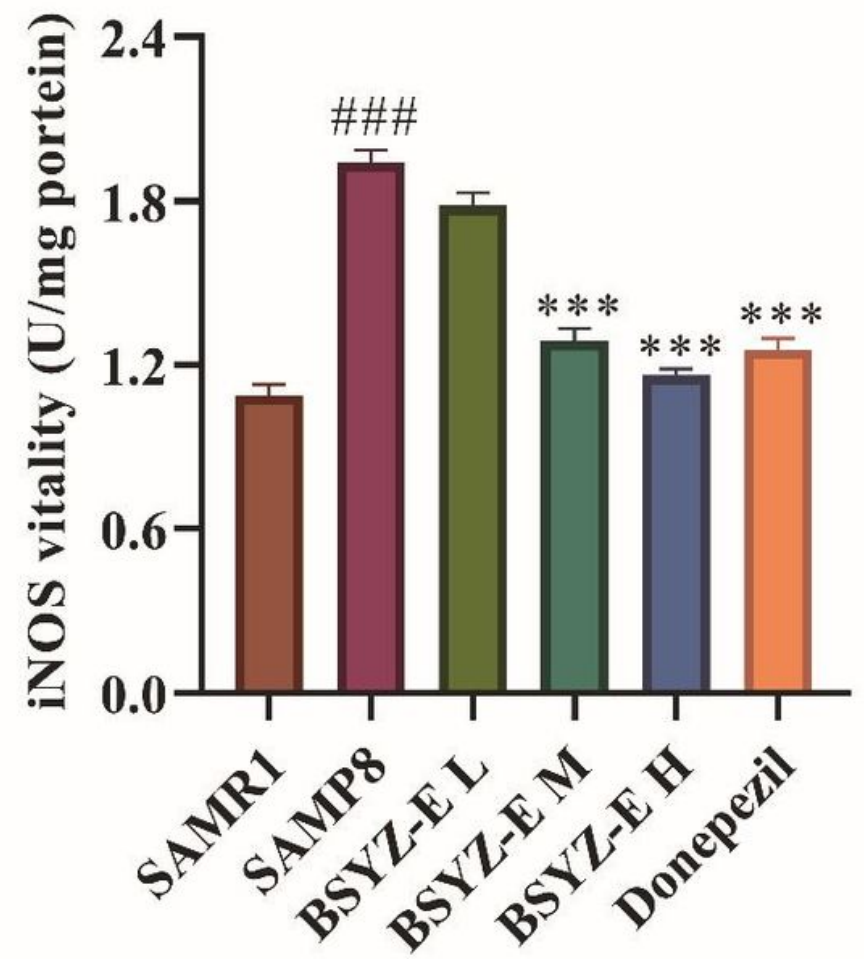

Figure 3

BSYZ-E ameliorates nitrosative stress in the hippocampus of SAMP8 mice. (A) NO level, (B) iNOS vitality were measured in the hippocampus. BSYZ-E L (1.46g/kg/day); BSYZ-E M (2.92 g/kg/day); BSYZ-E H $(5.84 \mathrm{~g} / \mathrm{kg} /$ day $)$. Experimental values were expressed as means $\pm S E M . \# p<0.05, \# \# p<0.01$ versus SAMR 1 group. ${ }^{*} \mathrm{p}<0.05,{ }^{* \star} \mathrm{p}<0.01,{ }^{\star \star *} \mathrm{p} \otimes 0.001$ versus SAMP8 group. 
A

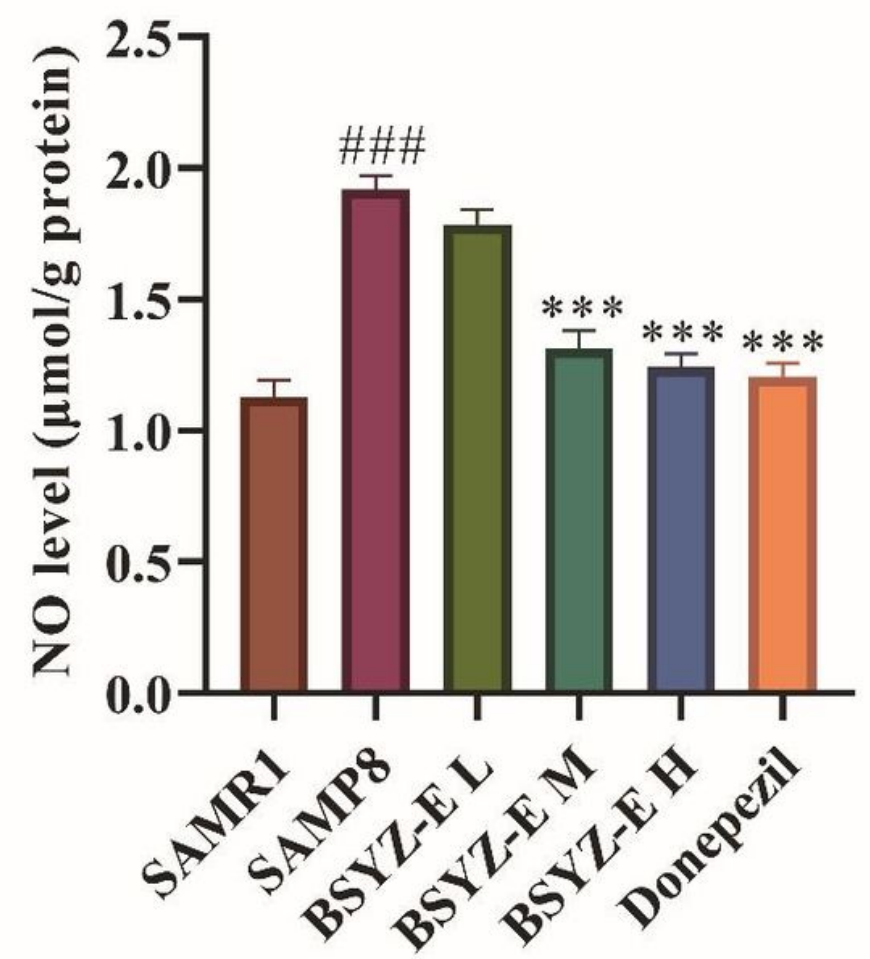

B

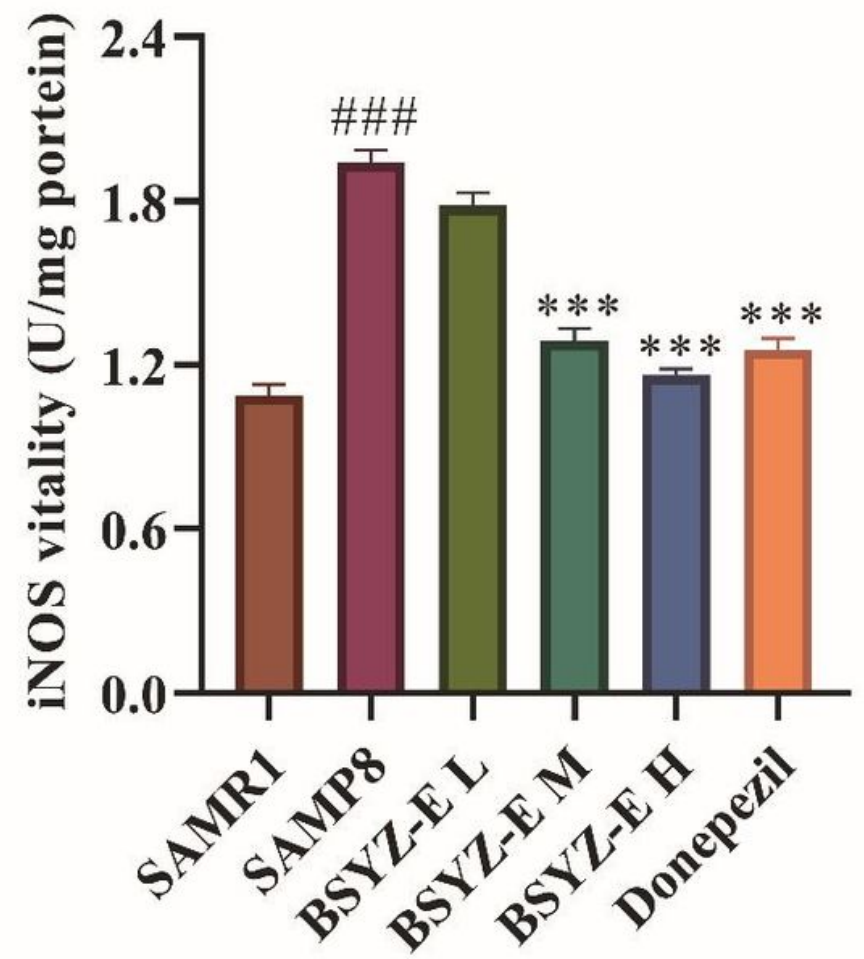

Figure 3

BSYZ-E ameliorates nitrosative stress in the hippocampus of SAMP8 mice. (A) NO level, (B) iNOS vitality were measured in the hippocampus. BSYZ-E L (1.46g/kg/day); BSYZ-E M (2.92 g/kg/day); BSYZ-E H $(5.84 \mathrm{~g} / \mathrm{kg} /$ day $)$. Experimental values were expressed as means $\pm S E M . \# p<0.05, \# \# p<0.01$ versus SAMR 1 group. ${ }^{*} \mathrm{p}<0.05,{ }^{* \star} \mathrm{p}<0.01,{ }^{\star \star *} \mathrm{p} \otimes 0.001$ versus SAMP8 group. 
$\mathbf{A}$
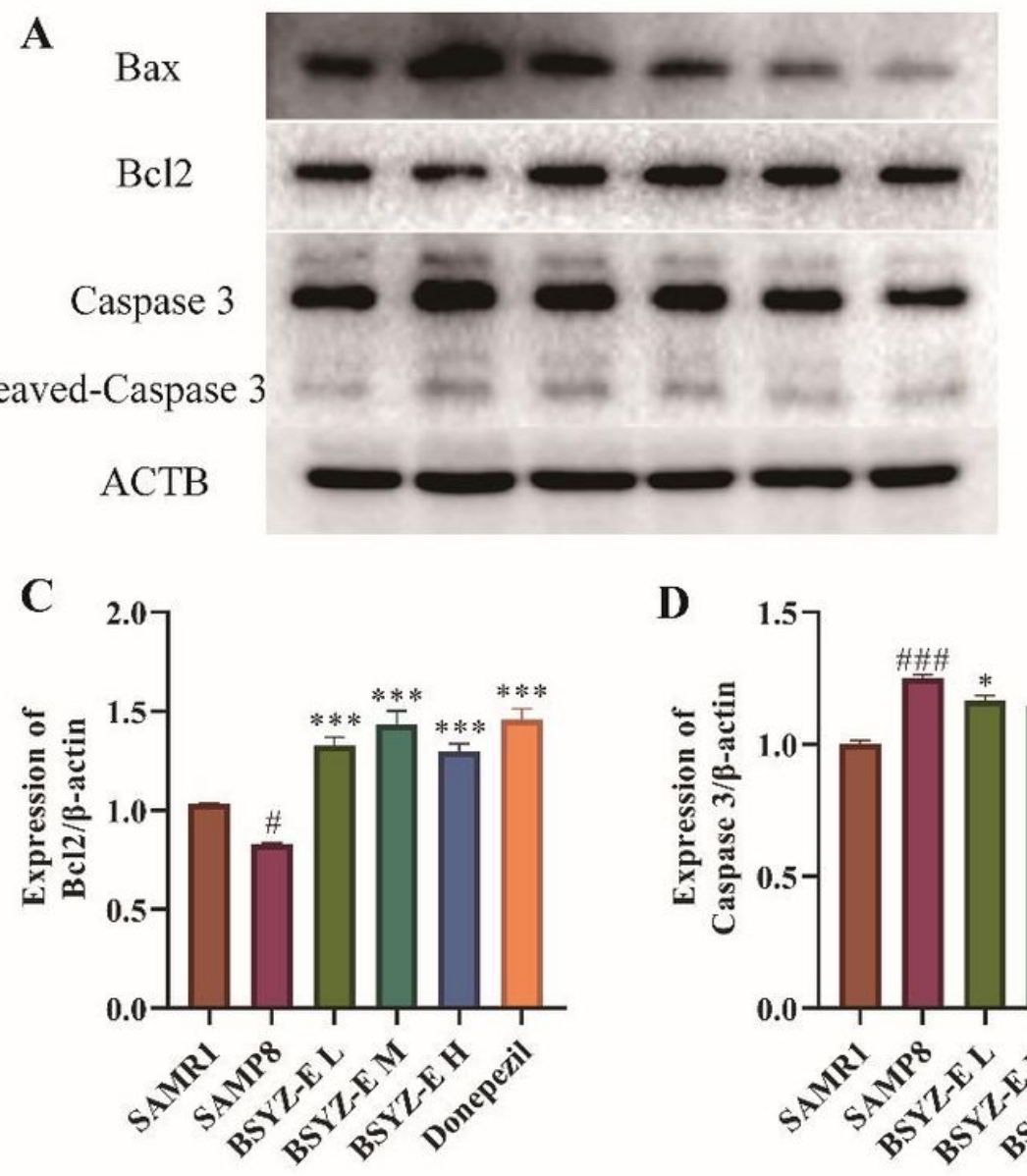

$22 \mathrm{kDa}$

$26 \mathrm{kDa}$

$35 \mathrm{kDa}$

$17 \mathrm{kDa}$

$43 \mathrm{kDa}$

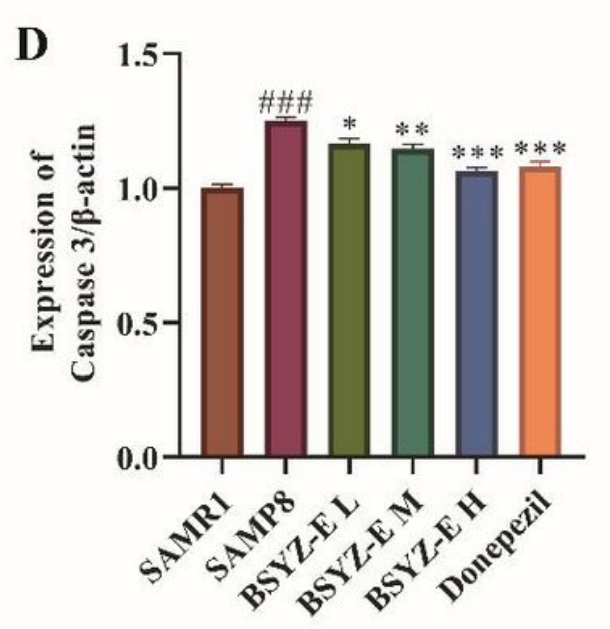

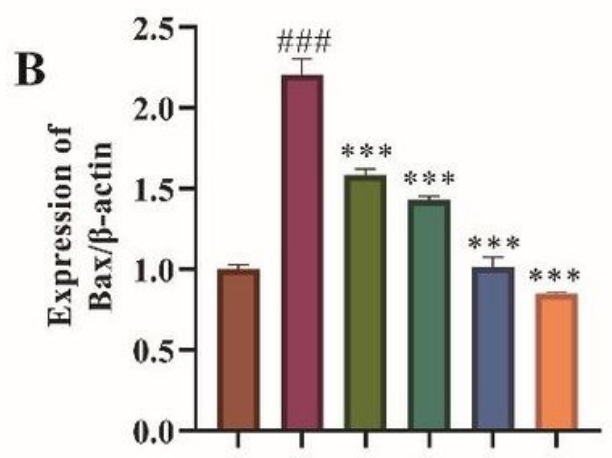

E

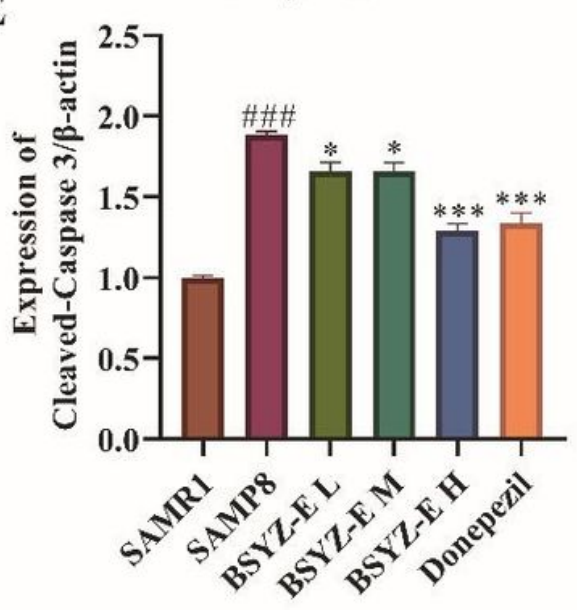

Figure 4

BSYZ-E ameliorates neuronal apoptosis in the hippocampus of SAMP8 mice. (A) The western blot of apoptosis proteins. (B) The expression of Bax in the hippocampus. (C) The expression of $\mathrm{Bcl}-2$ in the hippocampus. (D) The expression of Caspase-3 in the hippocampus. (E) The expression of CleavedCaspase-3 in the hippocampus. BSYZ-E L (1.46g/kg/day); BSYZ-E M $(2.92 \mathrm{~g} / \mathrm{kg} /$ day); BSYZ-E H (5.84 $\mathrm{g} / \mathrm{kg} /$ day). Experimental values were expressed as means $\pm S E M$. $\# p<0.05, \# \# p<0.01$ versus SAMR1 group. ${ }^{*} p<0.05,{ }^{* \star} p<0.01,{ }^{* \star *} \mathrm{p} \otimes 0.001$ versus SAMP8 group. 
A

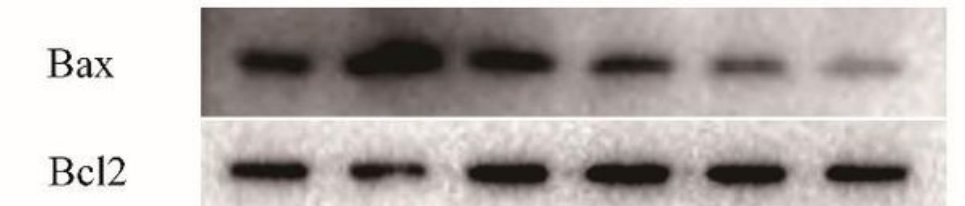

Caspase 3

Cleaved-Caspase 3

ACTB
$22 \mathrm{kDa}$

$26 \mathrm{kDa}$

$35 \mathrm{kDa}$

$17 \mathrm{kDa}$

$43 \mathrm{kDa}$

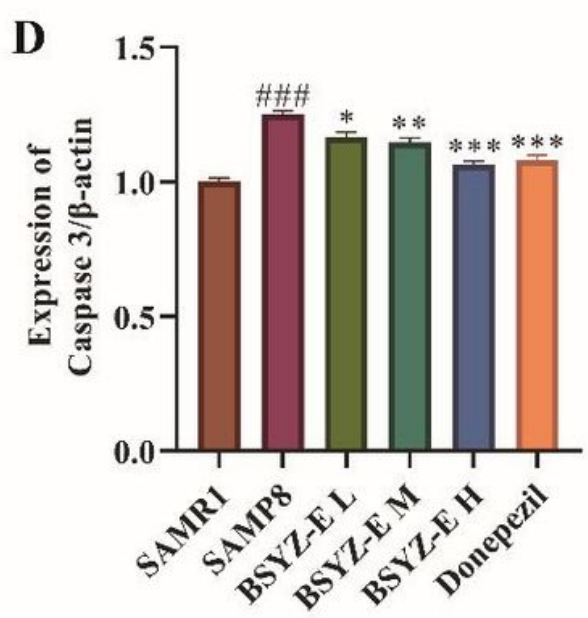

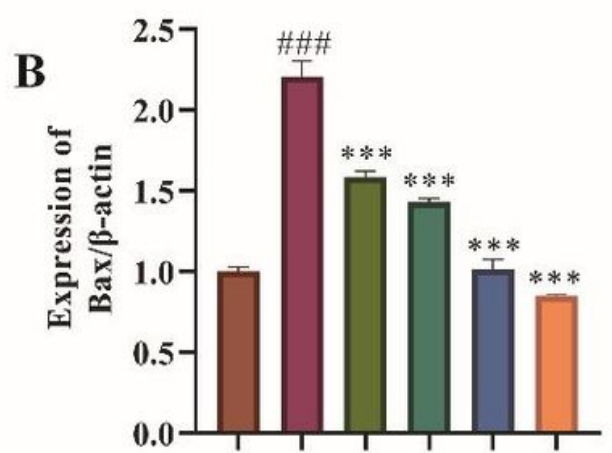

E
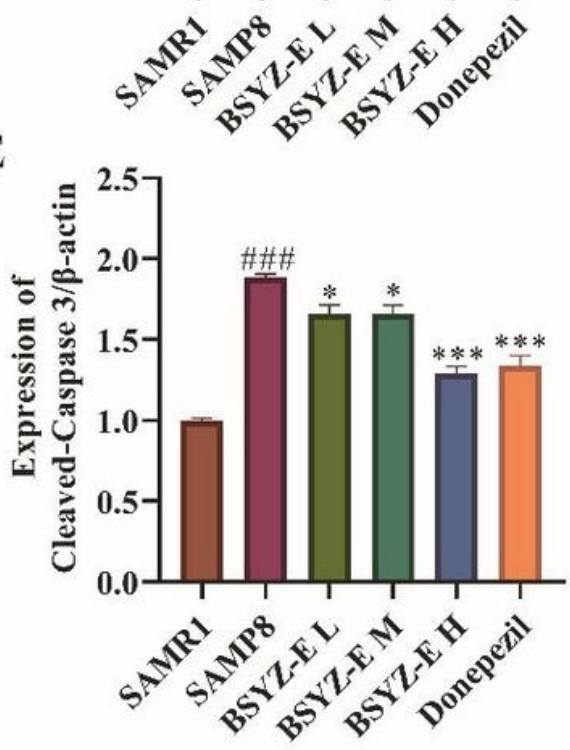

Figure 4

BSYZ-E ameliorates neuronal apoptosis in the hippocampus of SAMP8 mice. (A) The western blot of apoptosis proteins. (B) The expression of Bax in the hippocampus. (C) The expression of Bcl-2 in the hippocampus. (D) The expression of Caspase-3 in the hippocampus. (E) The expression of CleavedCaspase-3 in the hippocampus. BSYZ-E L (1.46g/kg/day); BSYZ-E M $(2.92 \mathrm{~g} / \mathrm{kg} /$ day); BSYZ-E H (5.84 $\mathrm{g} / \mathrm{kg} /$ day). Experimental values were expressed as means $\pm S E M$. $\# p<0.05, \# \# p<0.01$ versus SAMR1 group. ${ }^{*} \mathrm{p}<0.05,{ }^{* \star} \mathrm{p}<0.01,{ }^{* \star *} \mathrm{p} \otimes 0.001$ versus SAMP8 group. 
A

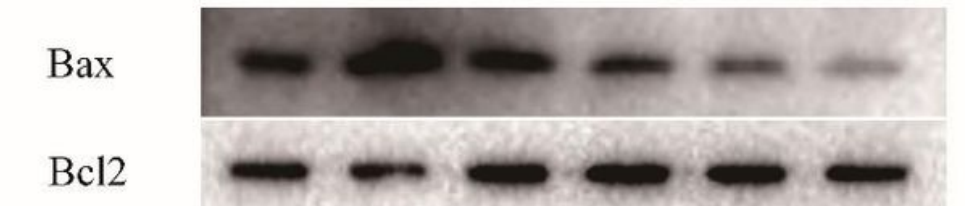

Caspase 3

Cleaved-Caspase 3

ACTB
$22 \mathrm{kDa}$

$26 \mathrm{kDa}$

$35 \mathrm{kDa}$

$17 \mathrm{kDa}$

$43 \mathrm{kDa}$

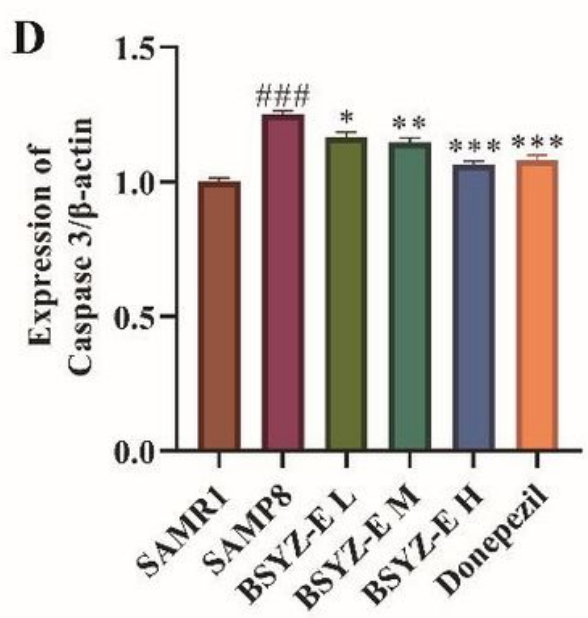

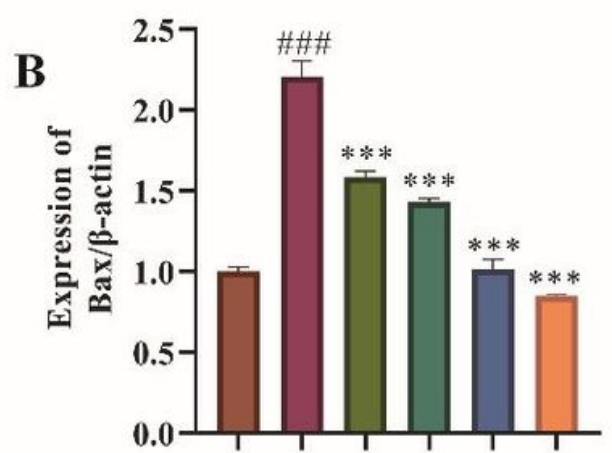

E
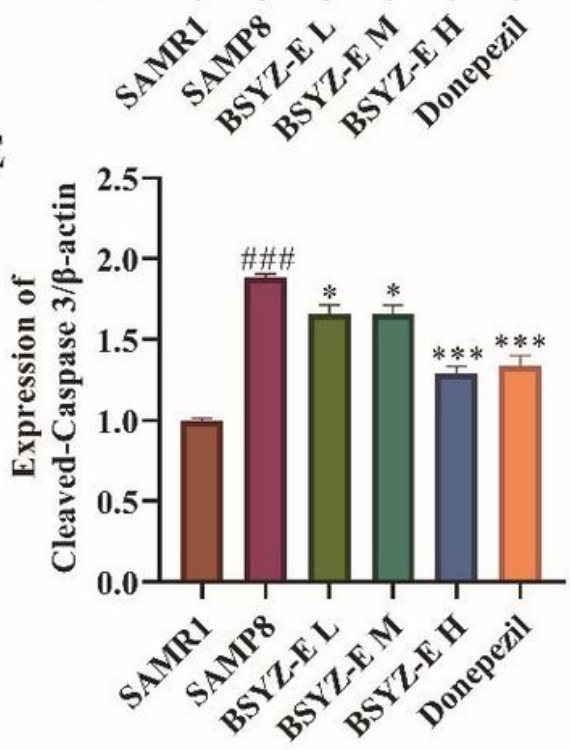

Figure 4

BSYZ-E ameliorates neuronal apoptosis in the hippocampus of SAMP8 mice. (A) The western blot of apoptosis proteins. (B) The expression of Bax in the hippocampus. (C) The expression of Bcl-2 in the hippocampus. (D) The expression of Caspase-3 in the hippocampus. (E) The expression of CleavedCaspase-3 in the hippocampus. BSYZ-E L (1.46g/kg/day); BSYZ-E M $(2.92 \mathrm{~g} / \mathrm{kg} /$ day); BSYZ-E H (5.84 $\mathrm{g} / \mathrm{kg} /$ day). Experimental values were expressed as means $\pm S E M$. $\# p<0.05, \# \# p<0.01$ versus SAMR1 group. ${ }^{*} \mathrm{p}<0.05,{ }^{* \star} \mathrm{p}<0.01,{ }^{* \star *} \mathrm{p} \otimes 0.001$ versus SAMP8 group. 

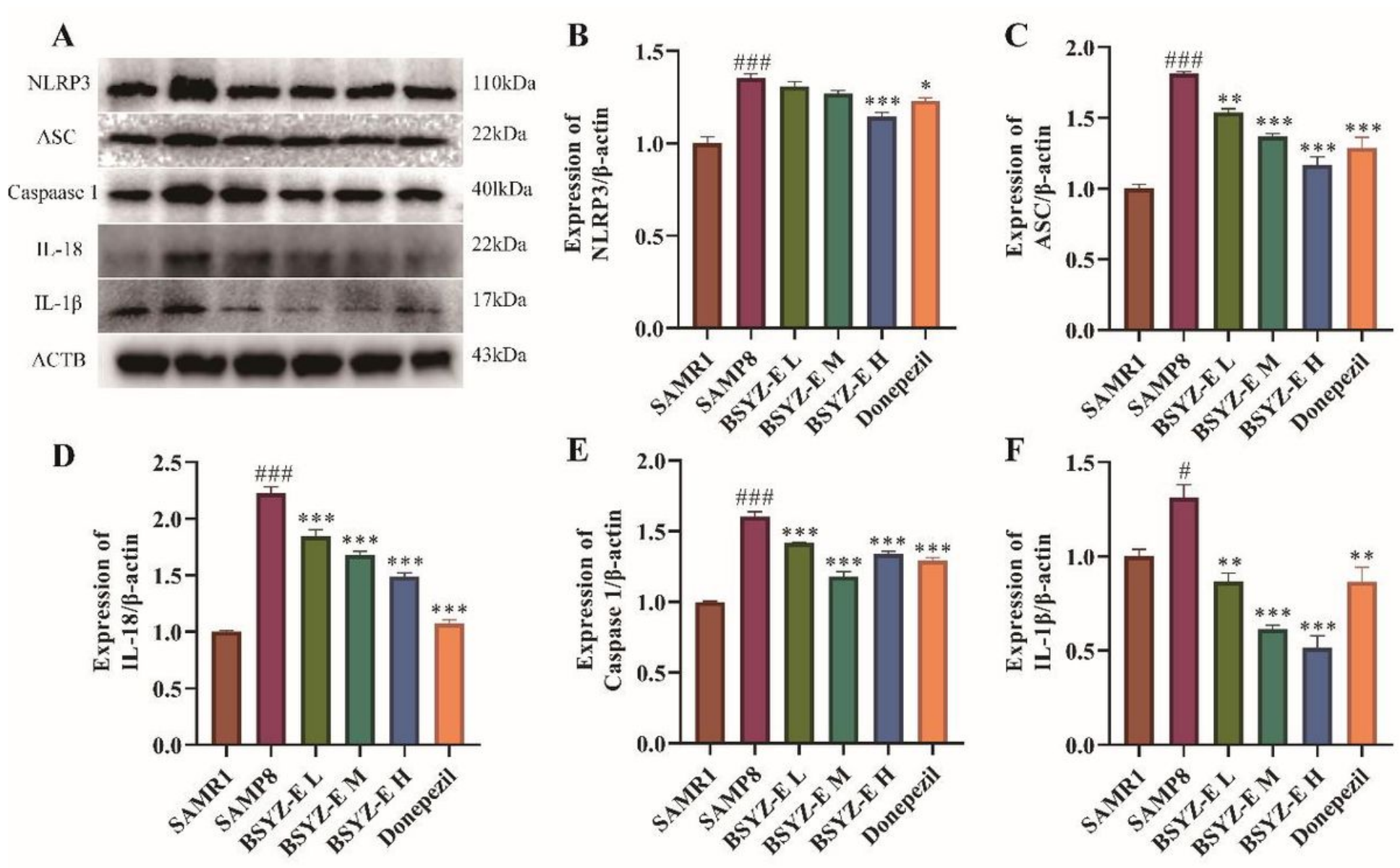

Figure 5

. BSYZ-E inhibits NLRP3 inflammasome activation in the hippocampus of SAMP8 mice. (A) The western blot of inflammation proteins. (B) The expression of NLRP3 in the hippocampus. (C) The expression of ASC in the hippocampus. (D) The expression of IL-18 in the hippocampus. (E) The expression of Caspase-1 in the hippocampus. (F) The expression of IL-1 $\beta$ in the hippocampus. BSYZ-E L (1.46g/kg/day); BSYZ-E M (2.92 g/kg/day); BSYZ-E H (5.84 g/kg/day). Experimental values were

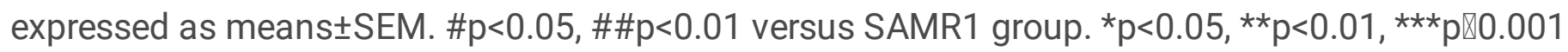
versus SAMP8 group. 

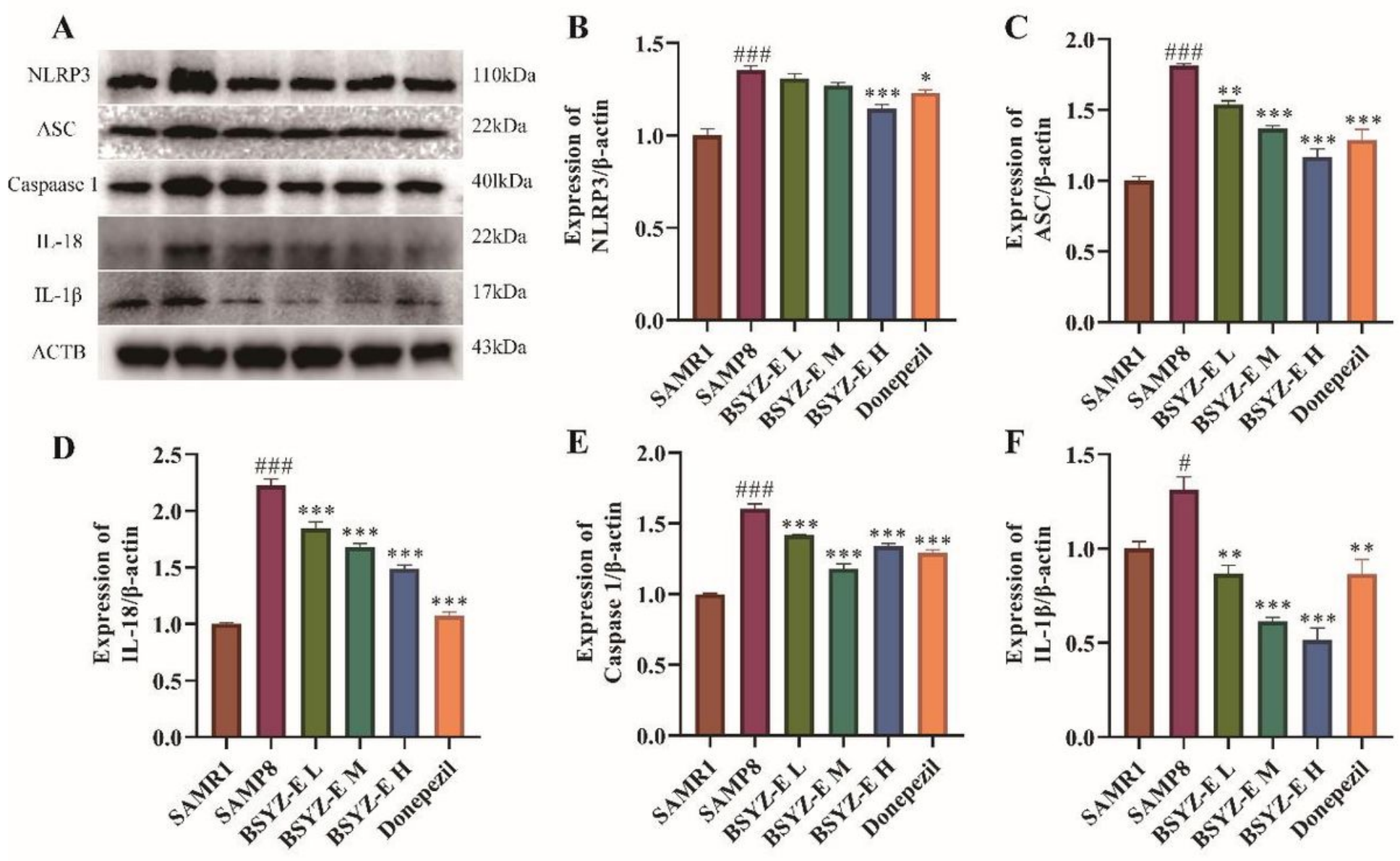

Figure 5

. BSYZ-E inhibits NLRP3 inflammasome activation in the hippocampus of SAMP8 mice. (A) The western blot of inflammation proteins. (B) The expression of NLRP3 in the hippocampus. (C) The expression of ASC in the hippocampus. (D) The expression of IL-18 in the hippocampus. (E) The expression of Caspase-1 in the hippocampus. (F) The expression of IL-1 $\beta$ in the hippocampus. BSYZ-E L (1.46g/kg/day); BSYZ-E M (2.92 g/kg/day); BSYZ-E H (5.84 g/kg/day). Experimental values were

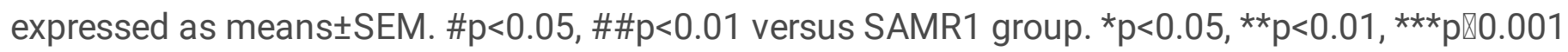
versus SAMP8 group. 

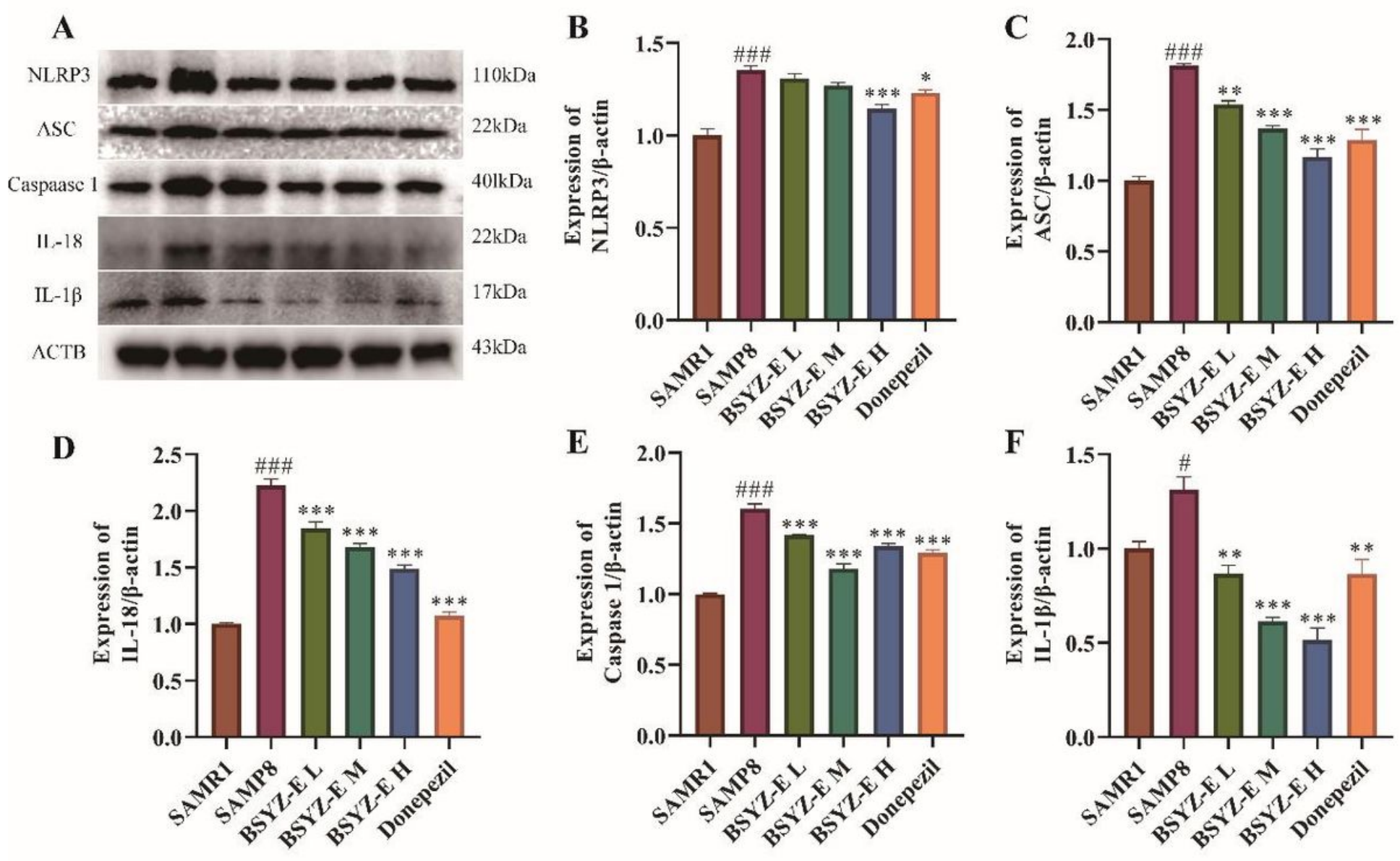

Figure 5

. BSYZ-E inhibits NLRP3 inflammasome activation in the hippocampus of SAMP8 mice. (A) The western blot of inflammation proteins. (B) The expression of NLRP3 in the hippocampus. (C) The expression of ASC in the hippocampus. (D) The expression of IL-18 in the hippocampus. (E) The expression of Caspase-1 in the hippocampus. (F) The expression of IL-1 $\beta$ in the hippocampus. BSYZ-E L (1.46g/kg/day); BSYZ-E M (2.92 g/kg/day); BSYZ-E H (5.84 g/kg/day). Experimental values were

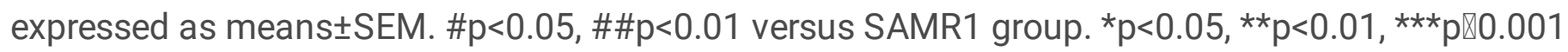
versus SAMP8 group. 